\title{
Inhibition of autophagy, lysosome and VCP function impairs stress granule assembly
}

\author{
SJ Seguin ${ }^{1}$, FF Morelli ${ }^{1}$, J Vinet ${ }^{1}$, D Amore ${ }^{1}$, S De Biasi ${ }^{2}$, A Poletti ${ }^{3}$, DC Rubinsztein ${ }^{4}$ and S Carra*,1
}

Stress granules (SGs) are mRNA-protein aggregates induced during stress, which accumulate in many neurodegenerative diseases. Previously, the autophagy-lysosome pathway and valosin-containing protein (VCP), key players of the protein quality control (PQC), were shown to regulate SG degradation. This is consistent with the idea that PQC may survey and/or assist SG dynamics. However, despite these observations, it is currently unknown whether the PQC actively participates in SG assembly. Here, we describe that inhibition of autophagy, lysosomes and VCP causes defective SG formation after induction. Silencing the VCP co-factors UFD1L and PLAA, which degrade defective ribosomal products (DRIPs) and 60S ribosomes, also impaired SG assembly. Intriguingly, DRIPs and 60S, which are released from disassembling polysomes and are normally excluded from SGs, were significantly retained within SGs in cells with impaired autophagy, lysosome or VCP function. Our results suggest that deregulated autophagy, lysosomal or VCP activities, which occur in several neurodegenerative (VCP-associated) diseases, may alter SG morphology and composition.

Cell Death and Differentiation (2014) 21, 1838-1851; doi:10.1038/cdd.2014.103; published online 18 July 2014

Cells respond to stresses, like heat shock or oxidative agents, which lead to protein aggregation, by activating the protein quality control $(\mathrm{PQC})$ and attenuating translation. ${ }^{1}$ The $\mathrm{PQC}$ consists of molecular chaperones and degradation systems and is an essential player of the proteotoxic stress response. To minimize protein aggregation, chaperones assist protein folding; when this is not effective, chaperones assist in targeting damaged substrates for clearance by the ubiquitin-proteasome system (UPS) and the lysosome-based degradation systems. ${ }^{2,3}$ In parallel, polysomes disassemble, releasing ribosomes, mRNAs, defective ribosomal products (DRIPs) and newly synthesized proteins, which, due to the stress, are prone to aggregation and are subjected to PQC and degradation. ${ }^{4}$

The mRNAs encoding 'housekeeping' proteins released from disassembling polysomes are sequestered into stress granules (SGs), non-membranous cytoplasmic foci where mRNAs are stored during stress. ${ }^{5}$ SGs have heterogeneous compositions and contain translationally silent mRNAs, early initiation factors, small, but not large, ribosomal subunits, mRNA-binding proteins, kinases and signaling molecules. ${ }^{5}$ Selective sequestration of these components within SGs occurs in a challenging subcellular environment where aggregate-prone substrates (released by polysomes) tend to accumulate. SG assembly is also triggered by the self-aggregation of RNA-binding proteins that contain prion-like domains, including T-cell-restricted intracellular antigen-1 (TIA-1). ${ }^{6}$ Unlike prionogenic fibrillar aggregates, SGs are dynamic structures, which disassemble within few hours after their formation.

Due to the heterogeneous composition of SGs and to the crowded molecular environment, SGs may, indirectly, require $\mathrm{PQC}$ assistance for proper assembly and disassembly. A number of $S G$ components have a role in $P Q C$, including ubiquitin and E3 ubiquitin ligases (TNF receptor-associated factor 2 and Roquin), ${ }^{7-10}$ while proteasome inhibition induces SGs. ${ }^{11}$ Histone deacetylase 6 (HDAC6), another SG component, ${ }^{8}$ facilitates the clearance of misfolded ubiquitinated proteins and participates in their targeting to the aggresome, a perinuclear structure that forms in response to an overload of $u n /$ misfolded proteins and enhances the degradation of toxic proteins. ${ }^{12}$ Moreover, HDAC6 binds to another SG component, Ras-GTPase-activating protein $\mathrm{SH} 3$ domainbinding protein (G3BP), which modulates the de-ubiquitinating enzyme ubiquitin specific peptidase 10 (USP10), which is also required for $S G$ formation. ${ }^{13,14}$ Although the exact role of these PQC components in SG dynamics is only partly understood, these findings suggest that $P Q C$ and $S G$ s are interconnected systems. SGs are degraded via macroautophagy (which we call autophagy) via a mechanism requiring the ubiquitin-selective chaperone valosin-containing protein (VCP). ${ }^{15}$ VCP modulates the ubiquitin-dependent proteolysis of selective clients by proteasome, ER-associated

\footnotetext{
${ }^{1}$ Dipartimento di Scienze Biomediche, Metaboliche e Neuroscienze, Universita' di Modena e Reggio Emilia, Modena, Italy; ${ }^{2}$ Dipartimento Chirurgico, Medico, Odontoiatrico e di Scienze Morfologiche, Universita' di Modena e Reggio Emilia, Modena, Italy; ${ }^{3}$ Dipartimento di Scienze Farmacologiche e Biomolecolari (DiSFeB), Universita' di Milano, Milan, Italy and ${ }^{4}$ Department of Medical Genetics, Cambridge Institute for Medical Research, University of Cambridge, Hills Road, Cambridge, UK *Corresponding author: S Carra, Dipartimento di Scienze Biomediche, Metaboliche e Neuroscienze, Universita' di Modena e Reggio Emilia, 41125 Modena, via Giuseppe Campi 287, Modena 41125, Italy. Tel: +39 0592055 265; Fax: +39 0592055 363; E-mail: serena.carra@unimore.it

Abbreviations: ATG, autophagy gene; DRIPs, defective ribosomal products; elF2 $\alpha$, alpha subunit of translation initiation factor 2; G3BP, Ras-GTPase-activating protein SH3 domain-binding protein; HDAC6, histone deacetylase 6; Hsp70, 70 kd heat shock protein; LAMP2, lysosomal associated membrane protein-2; PARP, poly ADP ribose polymerase; PLAA, phospholipase A2-activating protein; PQC, protein quality control; RPL19, ribosomal protein L19; RPS6, ribosomal protein S6; SG, stress granules; SQSTM1, sequestosome 1; TIA-1, T-cell-restricted intracellular antigen-1; Ubxd8, ubiquitin-like-domain-containing protein Ubxd8; UFD1L, ubiquitin fusion degradation 1 like; UPS, ubiquitin-proteasome system; USP10, ubiquitin specific peptidase 10; VCP, valosin-containing protein; Eerl, eeyarestatin I; OP-puro, O-propargyl-puromycin; Bort., bortezomib; ROI, region-of-Interest; CLQ, chloroquine
}

Received 26.2.14; revised 05.6.14; accepted 06.6.14; Edited by S Kumar; published online 18.7.14 
a TIA-1

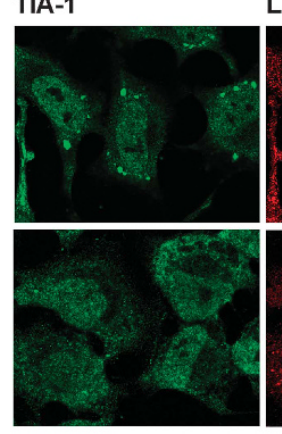

c

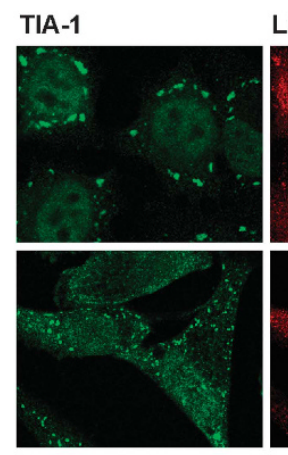

e TIA-1

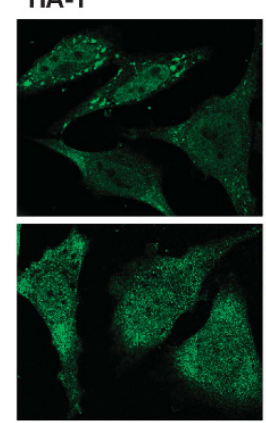

G3BP

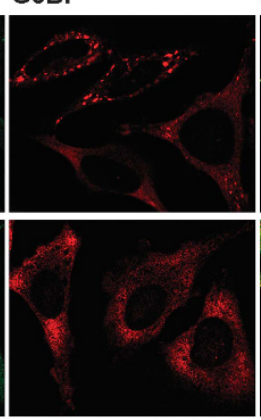

g

\section{TIA-1}

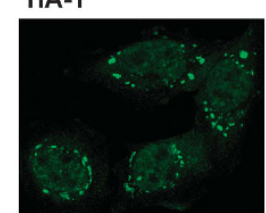

LC3

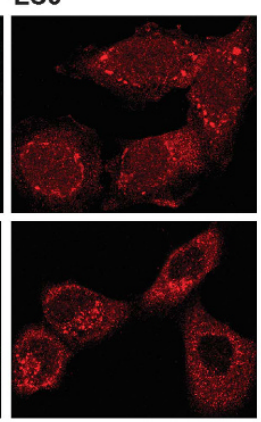

merge

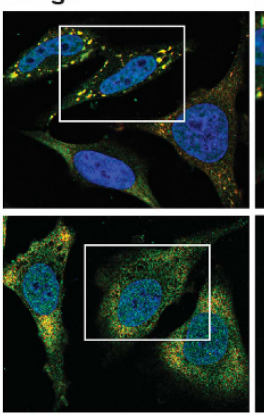

merge

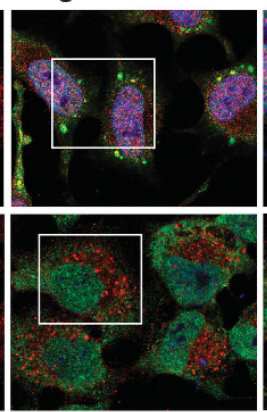

merge

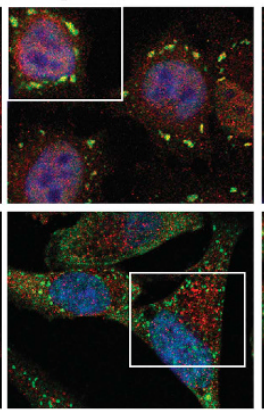

2.5x zoom

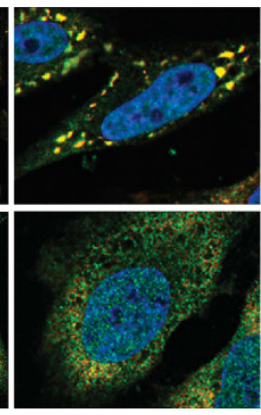

merge

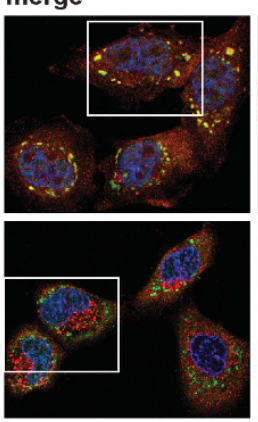

$2.5 \times$ zoom

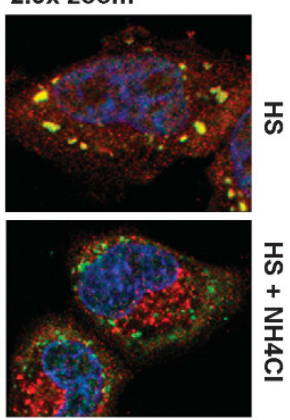

2.5x zoom

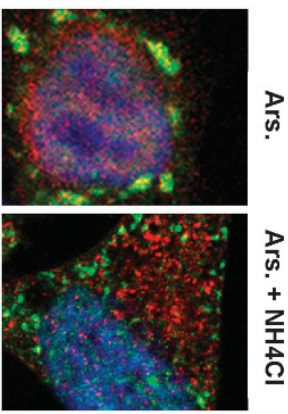

2.5x zoom

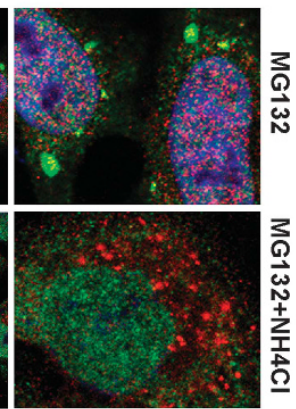

d

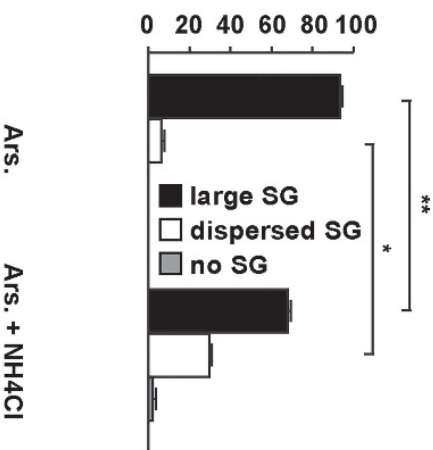

f

$\%$ cells with SG

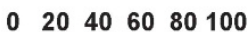

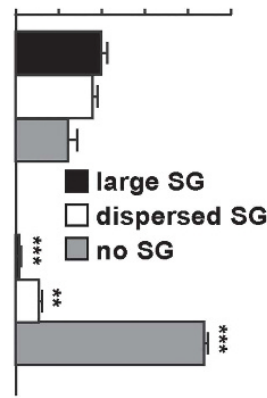

h \% cells with SG

$0 \quad 20406080100$

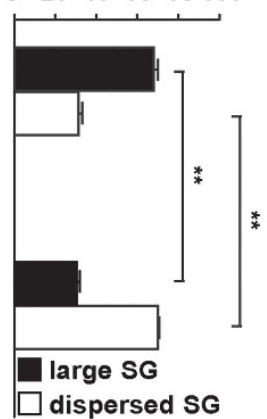

Figure 1 Lysosomotropic agents severely impair SG formation. HeLa cells treated for $3 \mathrm{~h}$ with MG132 alone or with ammonium chloride $\left(\mathrm{NH}_{4} \mathrm{Cl} ; \mathbf{a}, \mathbf{b}\right)$ or chloroquine (CLQ; b) were fixed and labeled with anti-TIA-1, LC3 and DAPI. (b) Percentage of cells with TIA-1-positive SGs is shown ( $\left.M=M G 132 ; N=N H_{4} C l ; C=C L Q\right)$. Error bar, S.E.M. ${ }^{* * *} P<0.001$ compared with MG132. HeLa cells were treated for $45 \mathrm{~min}$ with $0.5 \mathrm{mM}$ (c, d) or $0.1 \mathrm{mM}(\mathbf{e}, \mathbf{f})$ arsenite (Ars.); where indicated, cells were pretreated for $2 \mathrm{~h}$ 15 min with ammonium chloride $\left(\mathrm{NH}_{4} \mathrm{Cl}\right)$. Cells were fixed and labeled with anti-TIA-1, LC3 (c) or G3BP (e) and DAPI. (d, f) Percentage of cells with TIA-1-positive SGs (large or dispersed) and no SGs is shown. Error bar, S.E.M. (e) ${ }^{\star} P<0.05 ;{ }^{* \star} P<0.01 ;$ (f) ${ }^{* * \star} P<0.001 ;{ }^{* \star} P<0.01$ compared with Ars. $0.1 \mathrm{mM}$. (g, h) Hela cells pretreated or not with ammonium chloride $\left(\mathrm{NH}_{4} \mathrm{Cl}\right)$ for $2 \mathrm{~h} 15$ min were subjected to heat shock (HS) at $43.5^{\circ} \mathrm{C}$ for $45 \mathrm{~min}$, fixed and labeled with anti-TIA-1, LC3 and DAPI. (h) Quantitation of data in $\mathbf{g}$. Error bar, S.E.M. ${ }^{* *} P<0.01$. (a, c, e, g) $2.5 \times$ magnification of the selected area. See also Supplementary Figures S1, S2 and S3 
degradation and/or autophagosomes; ${ }^{16-18}$ this underscores the link between SGs and proteostasis. Here, we investigated whether impairment of PQC, autophagy and lysosomes affects SG assembly. We demonstrate that inhibition of VCP, autophagy or lysosomes affects SG formation, morphology and composition. a
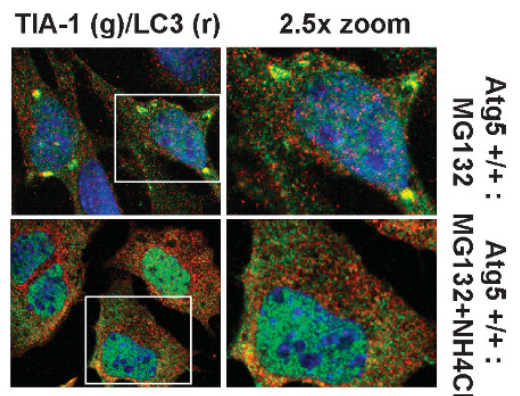

b
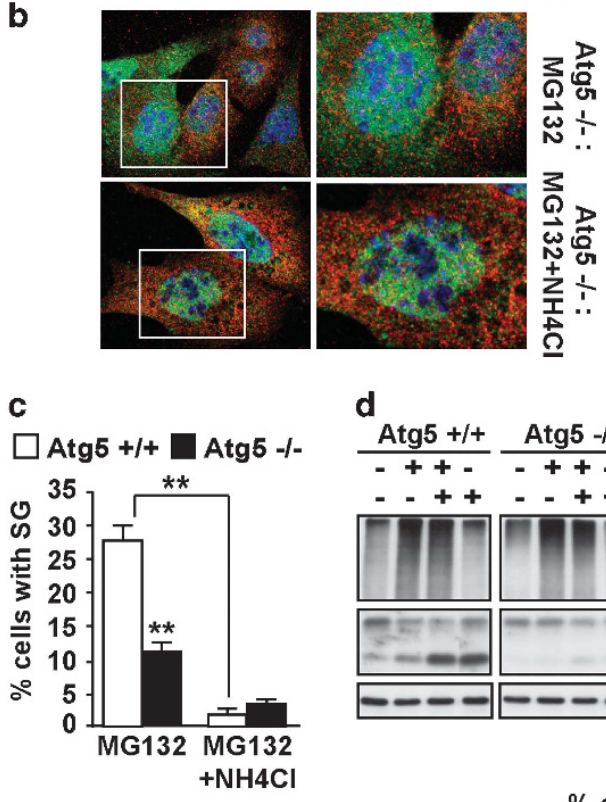

d

e

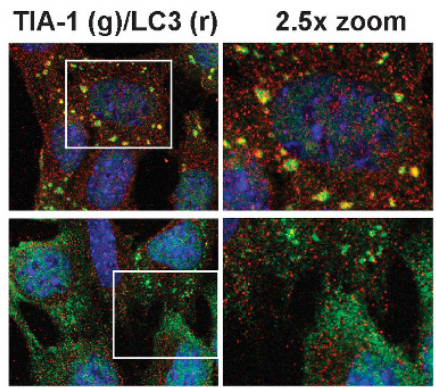

f
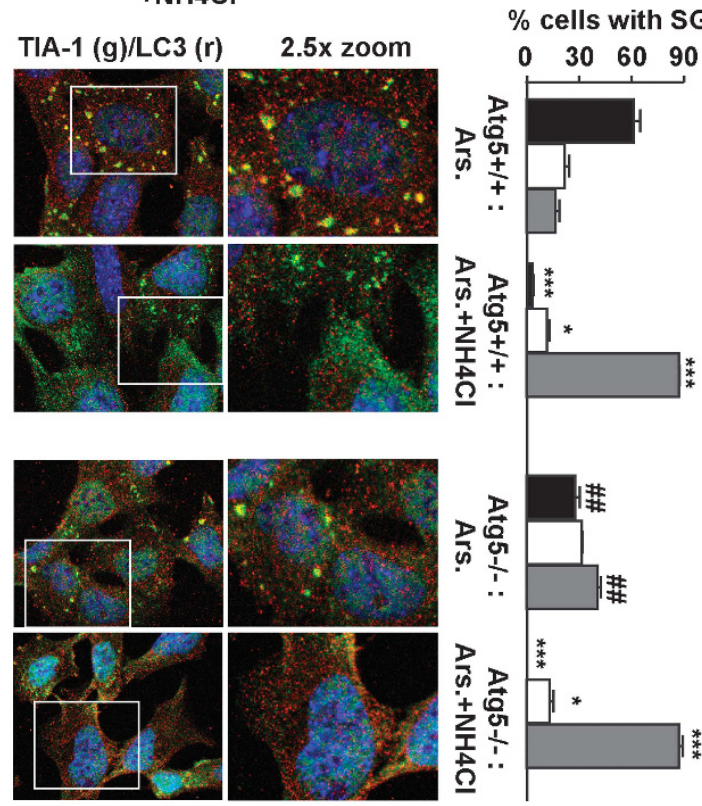

large SG $\square$ dispersed SG $\square$ no SG
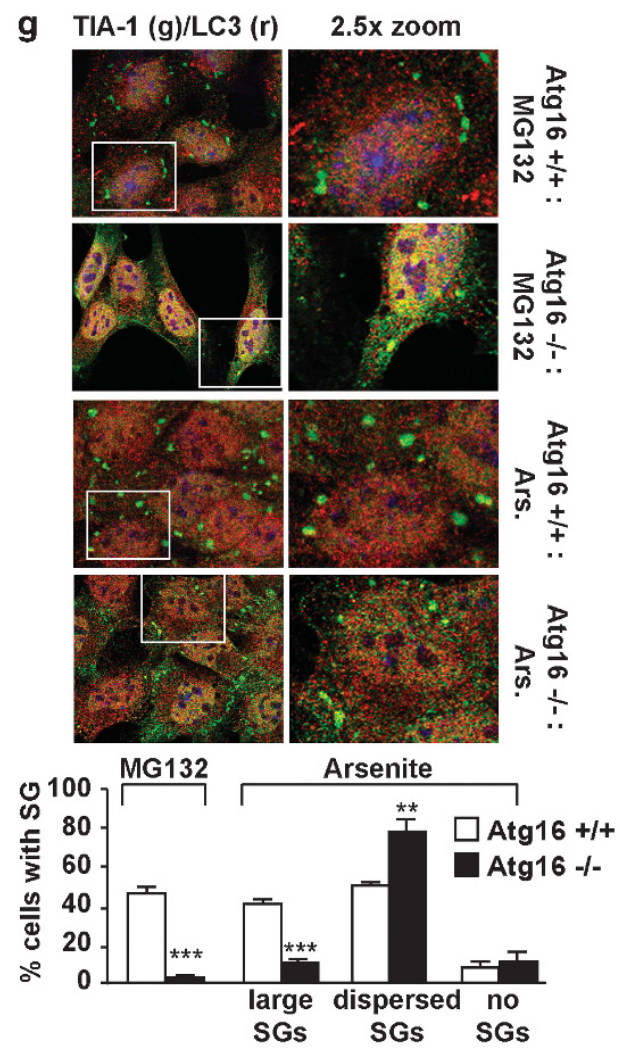

h

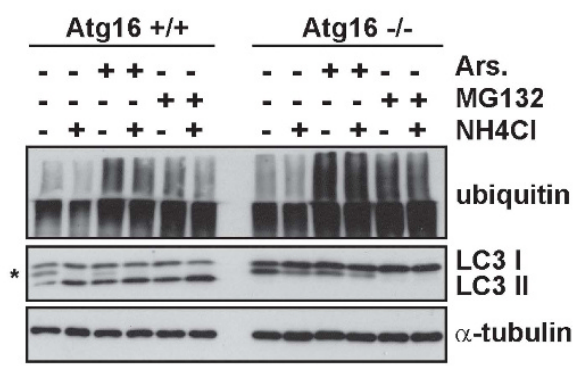

i

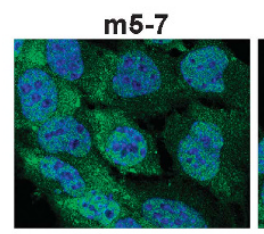

m5-7 + tet.

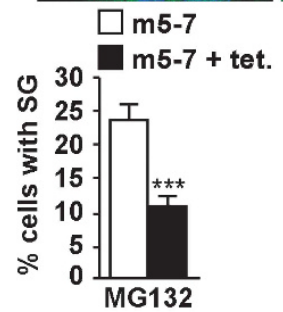




\section{Results}

Lysosomal inhibition impairs SG formation. To assess whether impairment of the PQC system may affect SG formation, we induced SGs using the proteasome inhibitor MG132 $2^{11}$ and simultaneously inhibited the lysosomes, the common endpoint for a number of degradation pathways. ${ }^{19,20}$ In line with previous reports, ${ }^{11}$ MG132 induced SGs in ca $70 \%$ of the cells, with a maximal peak after $3 \mathrm{~h}$; SGs, which are dynamic, disappeared after $8 \mathrm{~h}$ of MG132 treatment (Supplementary Figure S1A). Co-treatment of the cells with MG132 and the lysosomal inhibitors ammonium chloride $\left(\mathrm{NH}_{4} \mathrm{Cl}\right)$ or chloroquine (CLQ) suppressed MG132induced SGs (Figures $1 \mathrm{a}$ and $\mathrm{b}$ ). We saw no SGs even after $8 \mathrm{~h}$ co-treatment with $\mathrm{MG} 132$ and $\mathrm{NH}_{4} \mathrm{Cl}$ (Supplementary Figure S1A), which supports an inhibitory role, rather than a delay, on SG formation. MG132 caused the accumulation of ubiquitinated proteins, while $\mathrm{NH}_{4} \mathrm{Cl}$ increased levels of LC3$\mathrm{II}$, the autophagosome-associated, lipidated form of LC3 that accumulates if their lysosomal degradation is inhibited ${ }^{21}$ (Supplementary Figure S1A). When we monitored SGs and the efficacy of $\mathrm{NH}_{4} \mathrm{Cl}$, which causes a buildup of LC3-positive autophagosomes, by immunofluorescence using antibodies for TIA-1 and LC3, respectively, we observed LC3 recruitment to SGs (Figure 1a).

Since drugs that stabilize polysomes (cycloheximide) inhibit SG formation, ${ }^{22}$ we analyzed polysome distribution to test whether $\mathrm{NH}_{4} \mathrm{Cl}$ prevented polysome disassembly. $\mathrm{NH}_{4} \mathrm{Cl}$ did not alter the distribution of ribosomal protein S6 (RPS6), while addition of EDTA to pelleted polysomes dissociated them, as expected (Supplementary Figures S1B and C). MG132 alone, or with $\mathrm{NH}_{4} \mathrm{Cl}$, caused polysome disassembly, as no or only a minor RPS6 signal was detected throughout the gradient (Supplementary Figure $\mathrm{S} 1 \mathrm{C}$ ). Thus, $\mathrm{NH}_{4} \mathrm{Cl}$ prevents MG132-induced SGs by acting downstream of polysome disassembly.

Next, we tested if $\mathrm{NH}_{4} \mathrm{Cl}$ inhibited SG formation in response to arsenite, which also leads to protein aggregation. ${ }^{23}$ Arsenite $(0.5 \mathrm{mM})$ induced large SGs aligned in a ring-like shape in nearly all the cells (number of SGs/cell: $10.5 \pm 0.3$; SG size: $2.03 \mu \mathrm{M} \pm 0.05$; Figures $1 \mathrm{c}$ and $\mathrm{d}$ ). Pre-treatment with $\mathrm{NH}_{4} \mathrm{Cl}$ modified the number, size and distribution of SGs, which were more numerous, smaller (number of SGs/cell: 24.6 \pm 0.7 ; SG size: $0.94 \mu \mathrm{M} \pm 0.02$ ) and dispersed throughout the cytoplasm (Figures $1 \mathrm{c}$ and d). $\mathrm{NH}_{4} \mathrm{Cl}$ abrogated SG formation when we used lower concentrations of arsenite $(0.1 \mathrm{mM})$, which did not lead to a maximal $S G$ response (Figures 1e and f).
SGs form as a consequence of translation arrest (but are not required for translation arrest). ${ }^{24}$ Translational status was assessed using puromycin, which is incorporated into nascent peptides. $^{25}$ Puromycin-labeled proteins were detected in control cells and cells treated with $\mathrm{NH}_{4} \mathrm{Cl}$, but not after treatment with arsenite alone or combined with $\mathrm{NH}_{4} \mathrm{Cl}$ (Supplementary Figure S1D). In parallel, polysomes disassembled after arsenite and $\mathrm{NH}_{4} \mathrm{Cl}$ co-treatment (data not shown).

Assembly of SGs is triggered by the self-reversible aggregation of components that contain a prion-like domain, including TIA-1. ${ }^{6}$ We excluded that the small and dispersed structures that form in cells co-treated with arsenite and $\mathrm{NH}_{4} \mathrm{Cl}$ are proteinaceous aggregates rather than bona fide SGs, since both G3BP, a well-known SG marker (Figure 1e), and RNA, labeled using SYTO RNASelect (Supplementary Figure S2A), colocalized with TIA-1 in these cells.

To generalize the inhibitory effect of $\mathrm{NH}_{4} \mathrm{Cl}$ on SGs, we induced SGs with heat shock; $\mathrm{NH}_{4} \mathrm{Cl}$ also decreased the formation of heat shock granules (Figures $1 \mathrm{~g}$ and $\mathrm{h}$ ). Then, to further test the importance of lysosomes in SG formation, we used different combinations of lysosomal protease inhibitors leupeptin, E64d and pepstatin A. While $\mathrm{NH}_{4} \mathrm{Cl}$ and CLQ inhibit the activity of all lysosomal acid hydrolases, these inhibitors selectively target the activity of specific classes of proteases. Pre-treatment with leupeptin and E64d or leupeptin, E64d and pepstatin A caused both LC3-II accumulation and reduced MG132-induced SGs (Supplementary Figure S2B). LC3 recruitment into SGs was found under all stress conditions tested and was confirmed using three LC3 antibodies (Supplementary Figure S2C; Figures 1a, c and g). To determine which form of LC3, cytosolic LC3-I or autophagosome-anchored LC3-II, is recruited into SGs, we used Atg5 (autophagy gene 5) knockout ( - / - ) MEFs; these cells lack the autophagy ATG5 gene required for LC3-I to LC3-II conversion (Figure 2d). ${ }^{26}$ Also in Atg5 ${ }^{-1}$ - MEFs, SGs were positive for LC3, suggesting that it is cytosolic LC3-I that is recruited into SGs and consistent with SGs being non-membranous foci (Supplementary Figure S2D). $\mathrm{NH}_{4} \mathrm{Cl}$ impaired SG formation in Atg5 $5^{-1-}$ and Atg5 $5^{+/+}$MEFs, generalizing its inhibitory effect to different cell lines (Figures 2a-f). After MG132 or arsenite treatments, the percentage of cells positive for SGs was significantly reduced in $\mathrm{Atg} 5^{-/-}$ MEFs, as compared with Atg5 ${ }^{+/+}$MEFs (Figures 2a-f), thereby suggesting a potential interplay between autophagy and SGs. We further tested this hypothesis by using MEFs lacking the ATG16 gene, which is essential for autophagosome formation, ${ }^{27}$ as evidenced by the lack of LC3-II in Atg16 ${ }^{-1-}$ cells (Figures $2 \mathrm{~g}$ and $\mathrm{h}$ ). Similar to $\mathrm{Atg}^{-1-}$ cells,

\footnotetext{
Figure 2 Atg5 and Atg16 null cells show impaired SG formation. (a-d) Atg $5^{+1+}$ or Atg $5^{-1}$ MEFs were left untreated or treated for $3 \mathrm{~h}$ with MG132 and/or ammonium chloride $\left(\mathrm{NH}_{4} \mathrm{Cl}\right.$ ) and either fixed and labeled with anti-TIA-1, LC3 and DAPI (a-c) or processed for western blot (d). (c) Quantitation of data in a and b. Error bar, S.E.M. ${ }^{* *} P<0.01$ compared with MG132 in Atg5 ${ }^{+1+}$. (e, f) Atg $5^{+/+}$and Atg $5^{-l-}$ MEFs were treated for 45 min with arsenite (Ars.); where indicated, MEFs were pretreated for $2 \mathrm{~h}$ $15 \mathrm{~min}$ with $20 \mathrm{mM}$ ammonium chloride $\left(\mathrm{NH}_{4} \mathrm{Cl}\right)$. Cells were fixed and labeled with anti-TIA-1, LC3 and DAPI. Quantitation of data is shown. Error bar, S.E.M. ${ }^{* \star \star} P<0.001$; ${ }^{*} P<0.05$ compared with Ars.; ${ }^{\# \#} P<0.01$ Ars-treated Atg $5^{-l-}$ compared with Atg $5^{+l+}$. (g) Atg16 $6^{+l+}$ and Atg $16^{-l-}$ MEFs were treated for $3 \mathrm{~h}$ with MG132 or for 45 min with Ars., fixed and labeled with anti-TIA-1, LC3 and DAPI. Quantitation of data is shown. Error bar, S.E.M. ${ }^{* *} P<0.001 ;{ }^{* \star} P<0.01$. (h) Atg16 ${ }^{+l+}$ and Atg16 ${ }^{-l-}$ MEFs were treated as described in a; where indicated cells were pretreated for $2 \mathrm{~h} 15$ min with ammonium chloride $\left(\mathrm{NH}_{4} \mathrm{Cl}\right.$ ), prior to lysis and western blot. (i) m5-7 cells were grown for 7 days without $(-)$ or with $(+)$ tetracycline $(500 \mathrm{ng} / \mathrm{ml})$, prior to addition of ammonium chloride $\left(\mathrm{NH}_{4} \mathrm{Cl}\right)$ or MG132 for $3 \mathrm{~h}$. Cells were processed for western blot or fixed and labeled with anti-TIA-1 and DAPI. Quantitation of data is shown. Error bar, S.E.M. ${ }^{* * *} P<0.001$ compared with MG132 condition. (h, i) ${ }^{*}$ likely corresponds to LC3 T. $(\mathbf{a}, \mathbf{b}, \mathbf{e}-\mathbf{g}) 2.5 \times$ magnification of the selected area
} 
Atg16 ${ }^{-/-}$cells showed impaired SG formation following MG132 or arsenite treatments (Figure 2g). However, $\mathrm{NH}_{4} \mathrm{Cl}$ has an additional inhibitory effect on SGs in $\mathrm{Atg} 5^{-1-}$ cells
(Figure 2f), suggesting that other macroautophagy-independent routes may contribute to SG formation. ${ }^{28}$ Finally, to confirm whether autophagy influences SG assembly, we used a

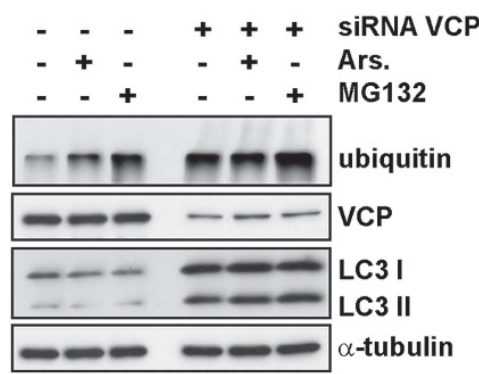

b TIA-1
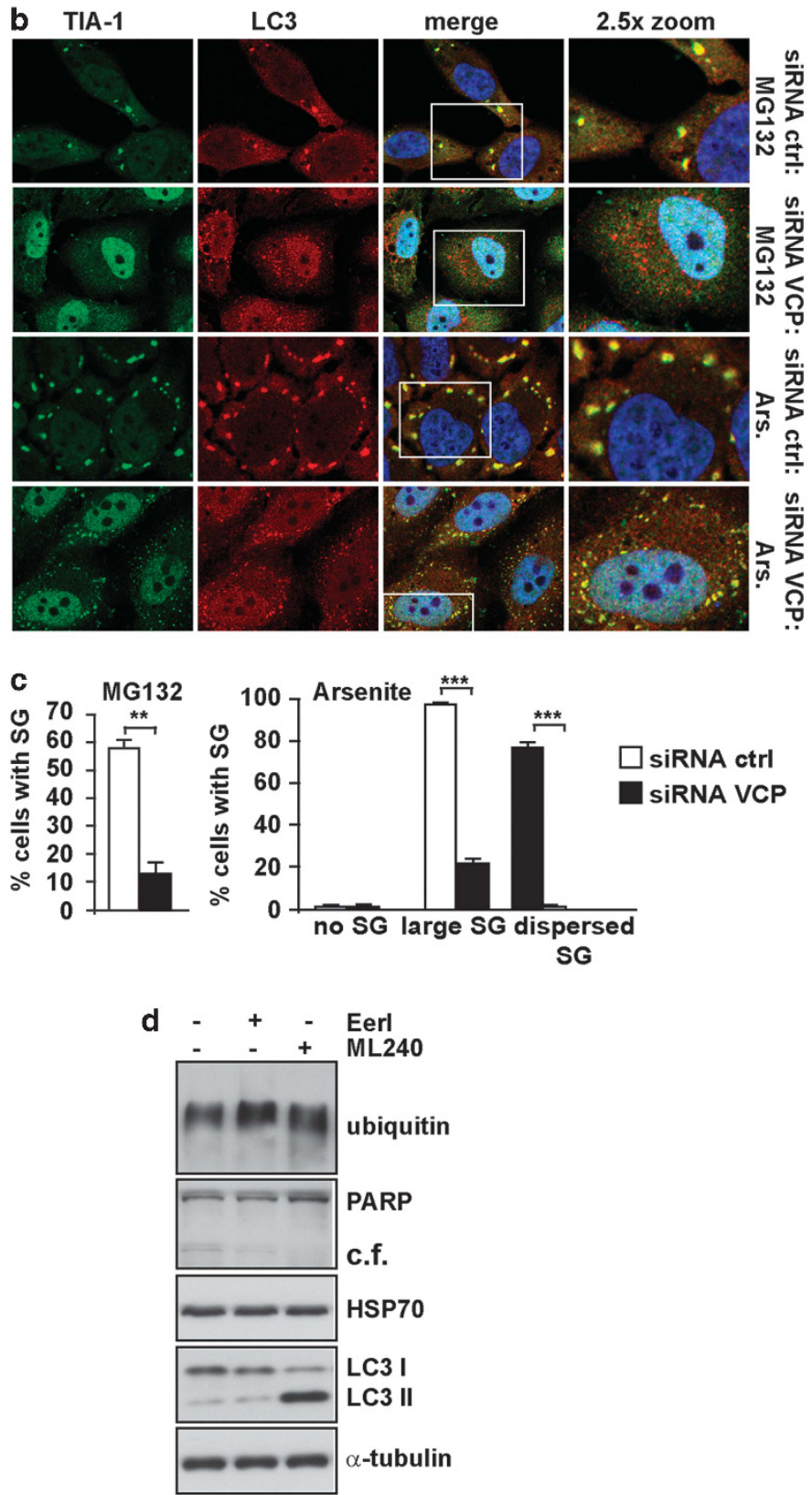

e
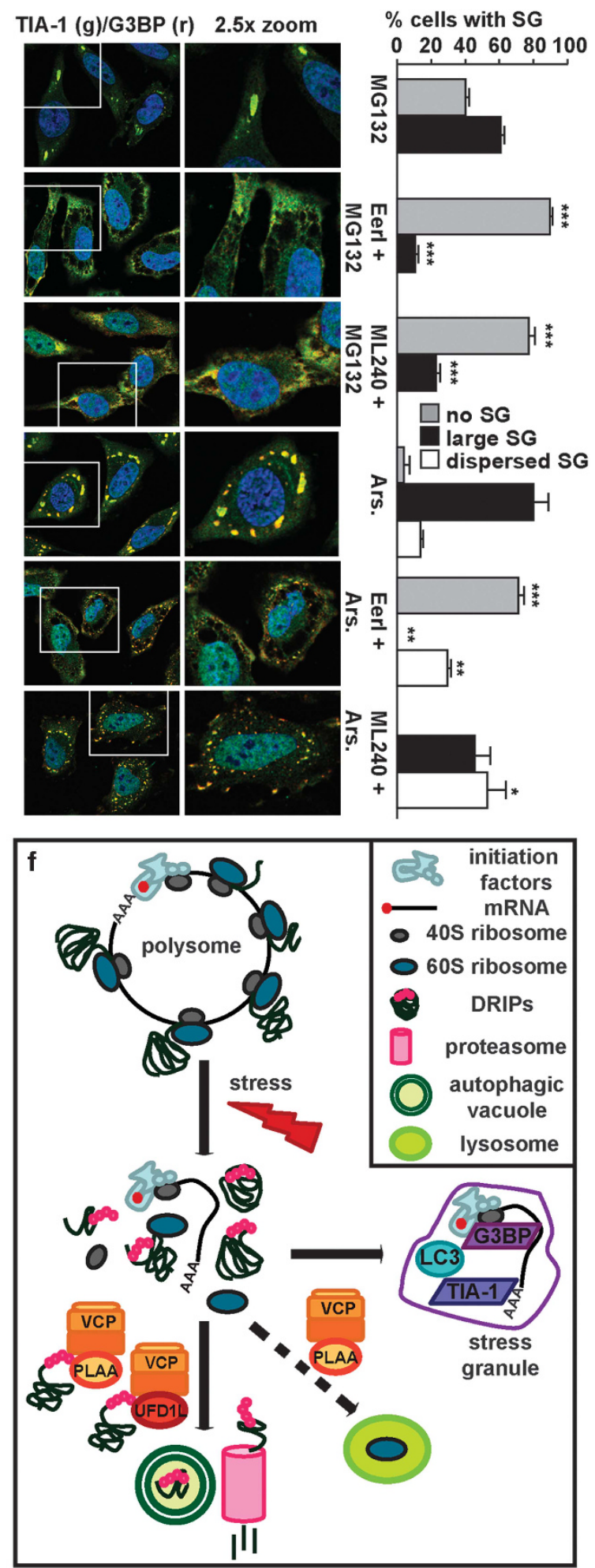
modified Atg5 ${ }^{-1-}$ MEFs that express an ATG5 transgene which is negatively regulated by tetracycline ( $\mathrm{m} 5-7)$, allowing inducible suppression of autophagy in the same cell line. ${ }^{29}$ Addition of tetracycline to the m5-7 cells suppressed LC3 lipidation (Figure 2i). MG132 treatment induced SGs in ca $25-30 \%$ of autophagy-competent m5-7 cells grown in the absence of tetracycline, similar to what was observed in Atg5 $5^{+/+}$MEFs (Figure 2, compare $\mathrm{c}$ and i). Addition of tetracycline to these m5-7 cells, which switches off autophagy/ATG5 expression, impaired MG132-induced SGs (Figure 2, compare $\mathrm{c}$ and i). Thus, inhibition of autophagy and lysosomes impairs SG assembly. This is complementary to recent findings demonstrating that SGs are cleared by autolysosomes and accumulate during the recovery, if autophagy is inhibited ${ }^{15}$ (a phenomenon we confirmed; Supplementary Figure S3A).

While $\mathrm{NH}_{4} \mathrm{Cl}$ (and CLQ) abrogated SG formation induced by MG132, they severely impaired (but did not block) SG formation by arsenite $(0.5 \mathrm{mM})$ or heat shock. We thus hypothesized that interplay between proteasome and lysosomes may exist and modulate SG assembly. To address this hypothesis, we treated HeLa cells with MG132 and arsenite $(0.5 \mathrm{mM})$, which together induce large $S G$ in all cells (Supplementary Figures S3B and C). Addition of $\mathrm{NH}_{4} \mathrm{Cl}$ inhibited SG assembly (Supplementary Figures S3B and C). Next, we used a low dose of Bortezomib, which inhibits the proteasome but does not induce SGs (Supplementary Figures S3D and E). Overnight pre-treatment with Bortezomib inhibited arsenite-induced SGs (Supplementary Figures S3D and E). These results support that interplay between proteasome and lysosomes is required for optimal SG assembly.

We thus asked how inhibition of the main protein degradation pathways may impair SG formation. While the $70 \mathrm{kd}$ heat shock protein (Hsp70) overexpression inhibits SG formation, ${ }^{11}$ we did not observe major induction of Hsp70 after short-term co-treatments with MG132 or arsenite and $\mathrm{NH}_{4} \mathrm{Cl}$ (Supplementary Figure S3F), or with overnight treatment with leupeptin, E64d and pepstatin A (Supplementary Figure S3G). Upregulation of Hsp70 was only found after overnight treatment with bortezomib (Supplementary Figure S3G). Thus, SG formation is not impaired by Hsp70 upregulation in our experimental conditions. Phosphorylation of the alpha subunit of translation initiation factor 2 (elF $2 \alpha$ ) is required for the induction of SGs upon arsenite, but not MG132. ${ }^{30}$ Arsenite alone or in combination with $\mathrm{NH}_{4} \mathrm{Cl}$ induced elF2 $\alpha$ phosphorylation (Supplementary Figure $\mathrm{S} 3 \mathrm{H}$ ), pointing to a different mechanism responsible for impaired SG assembly upon lysosome inhibition. We excluded the possibility that apoptosis may account for our observations, since pretreatment with the pan-caspase inhibitor zVAD-fmk could not rescue the inhibitory effect exerted by $\mathrm{NH}_{4} \mathrm{Cl}$ on $\mathrm{MG132}$ or arsenite-induced SGs (Supplementary Figure S3I), and the percentage of apoptotic and necrotic cells and cells with depolarized mitochondria were similar under all conditions tested both in HeLa (Supplementary Table S1) and autophagy-proficient/deficient cells (Supplementary Table S2).

Depletion of VCP impairs SG formation. VCP knockdown in HeLa cells led to accumulation of ubiquitinated proteins and LC3-II (Figure 3a) ${ }^{17}$ and decreased SG formation induced by MG132 and arsenite (Figures $3 b$ and $c$ ). The arsenite-induced SGs forming in VCP-deficient cells also contained LC3 (Figure 3b), supporting that LC3 recruitment within SGs is independent on VCP. Two well-known inhibitors of VCP, Eeyarestatin I (Eerl) and ML240 led to a mild accumulation of ubiquitinated proteins, while only ML240 also caused LC3-II buildup, in line with previous findings (Figure $3 d) ;{ }^{31}$ however, they did not induce poly ADP ribose polymerase (PARP) cleavage or Hsp70 (Figure 3d). Neither Eerl nor ML240 induced SGs (data not shown); instead, they impaired SG assembly following MG132 or arsenite treatment (Figure 3e), consistent with what we observed with VCP knockdown.

Prior to SG formation, polysomes disassemble and release ubiquitinated un/misfolded proteins, DRIPs and ribosomal subunits (Figure $3 f$ ). DRIPs, which represent ca the $30 \%$ of newly synthesized proteins, ${ }^{4}$ are aggregation-prone and may possibly affect the reversible self-aggregation of TIA-1, which drives SG formation. ${ }^{6}$ In addition, while the small ribosomal subunit (40S) is a component of SGs, the large one (60S) is excluded from and antagonistic to SGs. ${ }^{22,32}$ To enable client selection, VCP interacts with co-factors, like ubiquitin fusion degradation 1 like (UFD1L) and phospholipase A2-activating protein (PLAA). UFD1L promotes with VCP the degradation of DRIPs bound to translating ribosomes ${ }^{33}$ (Figure $3 f$ ). PLAA forms a complex that also contains HDAC6, a SG component. ${ }^{34}$ This complex modulates ubiquitin turnover and client transfer to HDAC6 for targeting to aggresome. ${ }^{34-36}$ VCP and PLAA are also required in yeast for ribophagy, the lysosomemediated degradation of $60 S^{37,38}$ (Figure $3 f$ ). We speculated that defective clearance of DRIPs and 605 mediated by lysosome/autophagy, proteasome and VCP (and co-factors), may contribute to impaired SG growth. In line with this hypothesis, knockdown of PLAA (Figures $4 \mathrm{a}-\mathrm{C}$ ) or UFD1L (Figures 4d-f) impaired MG132 and arsenite-induced SGs, although their effect was less severe than VCP knockdown.

Figure 3 Depletion and inhibition of VCP severely impair SG formation. (a-c) HeLa cells lipofected with control (ctrl) or VCP siRNA for $72 \mathrm{~h}$ were left untreated (data not shown) or treated for $3 \mathrm{~h}$ with MG132 or $45 \mathrm{~min}$ with arsenite (Ars.) Cells were lysed, processed for western blot (a) or fixed and labeled with anti-TIA-1, LC3 and DAPI (b). (c) Quantitation of data in b. Error bar, S.E.M. ${ }^{* *} P<0.01 ;{ }^{* *} P<0.001$. (d) HeLa cells not treated or treated for $3 \mathrm{~h}$ with ML240 or Eerl were lysed and processed for ubiquitin, PARP (c.f.: cleaved-fragment), Hsp70, LC3 and $\alpha$-tubulin western blot. (e) Cells were treated for $3 \mathrm{~h}$ with MG132 or for $45 \mathrm{~min}$ with Ars. alone or in combination with ML240 or Eerl; cells were fixed and labeled with anti-TIA-1, G3BP and DAPI. Quantitation of data is shown. Error bar, S.E.M. For MG132 co-treatments: ${ }^{* \star *} P<0.001$ compared with MG132 alone; for Ars. co-treatments: ${ }^{* \star \star} P<0.001$; ${ }^{* \star} P<0.01 ;{ }^{*} P<0.05$ compared with Ars. alone. (f) Schematic model of the known role of VCP in targeting DRIPs and large ribosome subunits (60S) to degradation. Polysomes are a cluster of ribosomes that synthesize nascent chains from mRNAs. Upon stress, including MG132 or arsenite treatments, polysomes disassemble and their components follow different fates. mRNAs together with initiation factors and the small (40S) ribosome subunit are sequestered into stress granules (SGs). DRIPs are ubiquitinated and routed to degradation. VCP bound to $60 S$ modulates the degradation of DRIPs. Co-factors such as UFD1L and PLAA assist VCP in client targeting to both proteasome and autophagy for clearance. In parallel, 60S are also released but are excluded from SGs and can become damaged under stress conditions (e.g., oxidation due to arsenite). 60S are targeted to lysosome for degradation, a process called ribophagy. VCP and PLAA are also required in yeast for ribophagy. (b, e, f) $2.5 \times$ magnification of the selected area. See also Supplementary Figure S4 


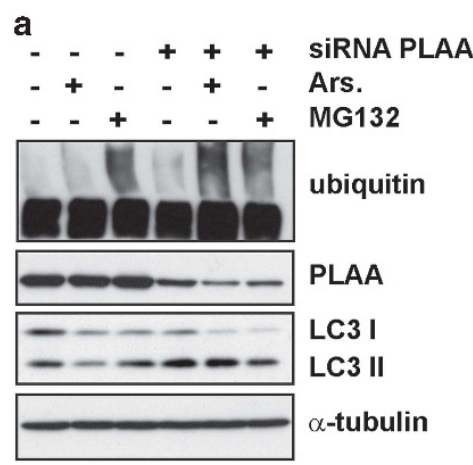

b

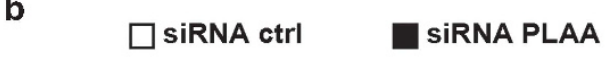

c
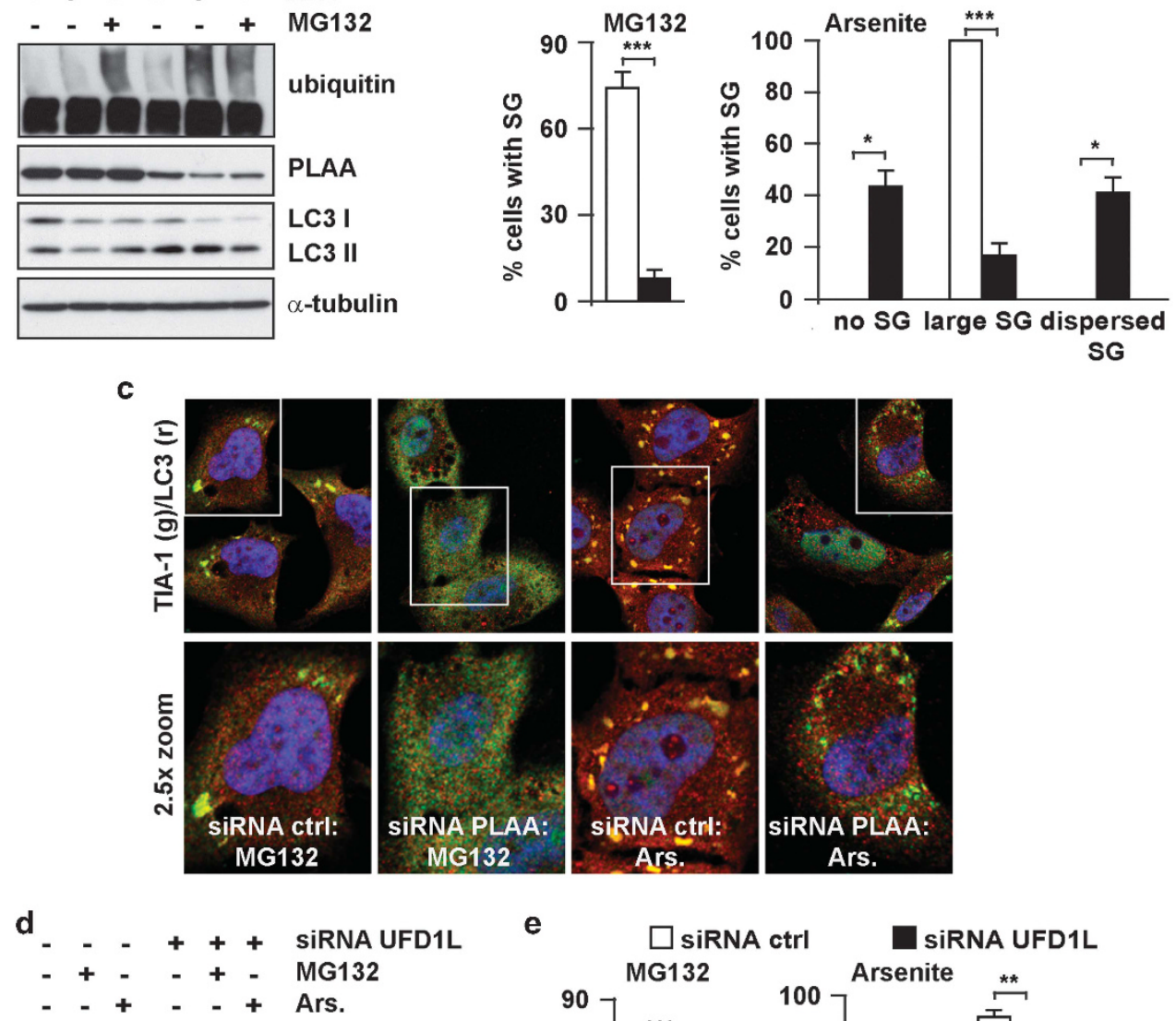

e
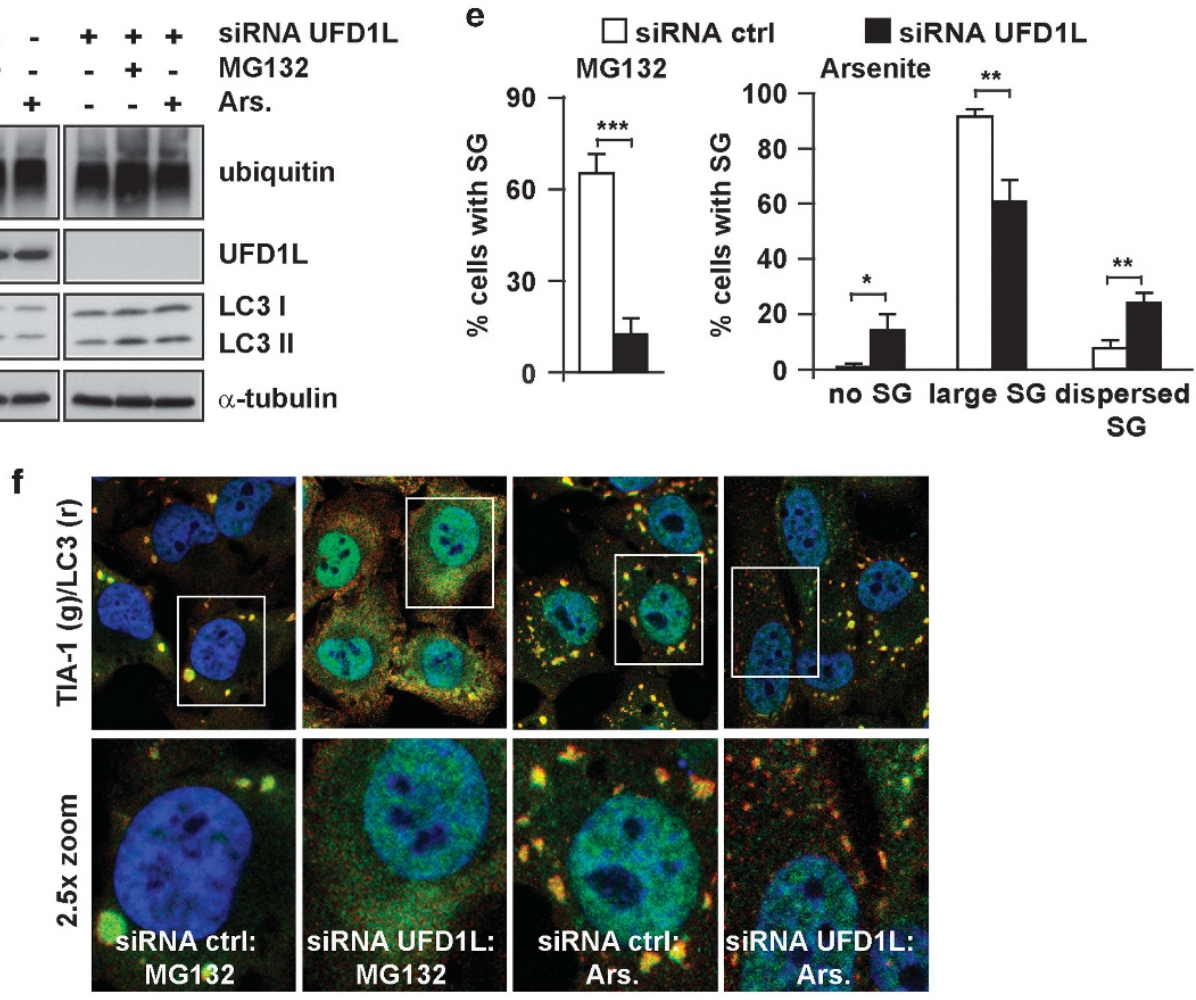

Figure 4 Depletion of the VCP co-factors PLAA and UFD1L affects SG formation. HeLa cells lipofected with control (ctrl), PLAA (a-c) or UFD1L siRNA (d-f) for $72 \mathrm{~h}$ were left untreated (data not shown) or treated for $3 \mathrm{~h}$ with MG132 or $45 \mathrm{~min}$ with arsenite (Ars). Cells were lysed and processed for western blot (a, d), or fixed and labeled with antiTIA-1, LC3 and DAPI (c, f). (b, e) Quantitation of data in $\mathbf{c}$ and f. Error bar, S.E.M. ${ }^{* \star} P<0.001 ;{ }^{* \star} P<0.01 ;{ }^{*} P<0.05$. (c, f) $2.5 \times$ magnification of the selected area. See also Supplementary Figure S4 
a

Alexa594-azide/TIA-1 2.5 x zoom
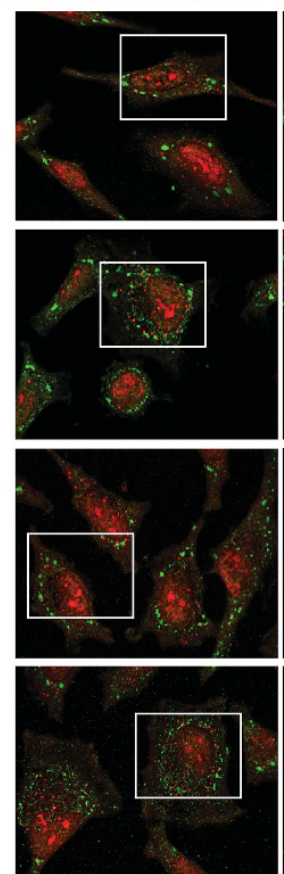

d
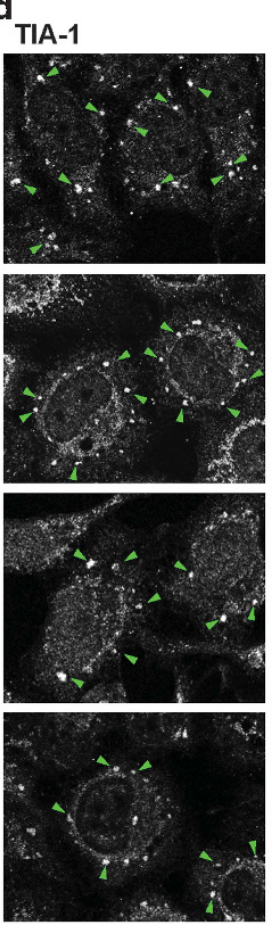

Arsenite
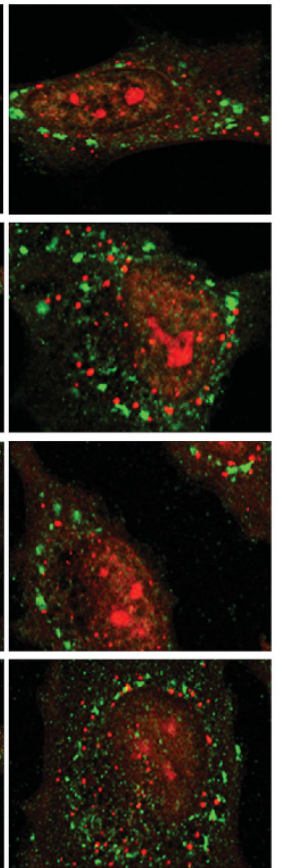

Alexa594-azide
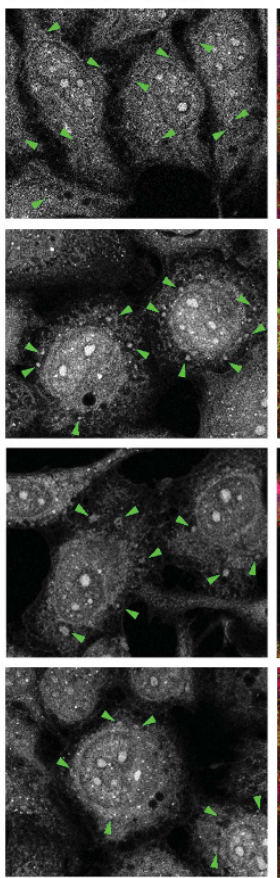

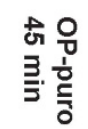

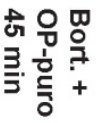

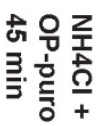

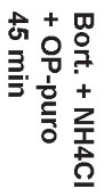
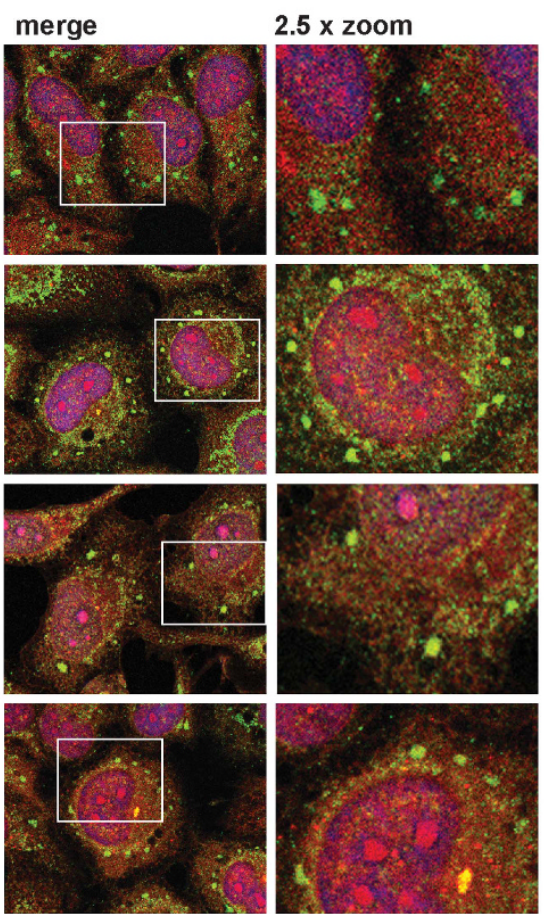

b
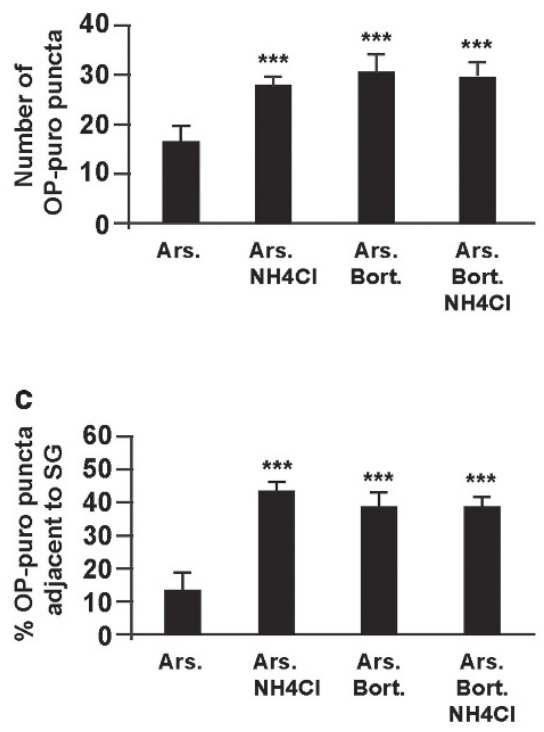

NH4CI

Figure 5 Inhibition of proteasome and lysosome or silencing of VCP and co-factors lead to the accumulation of OP-puro-labeled DRIPs adjacent to or within SGs. (a-c) HeLa cells were co-treated for 45 min with OP-puro and arsenite (Ars.); where indicated, cells were pretreated with bortezomib (Bort.) overnight and/or ammonium chloride $\left(\mathrm{NH}_{4} \mathrm{Cl}\right)$ for $2 \mathrm{~h} 15 \mathrm{~min}$. Cells were fixed and labeled with Alexa594-Azide and anti-TIA-1. (b) The number of OP-puro puncta per cell is shown. Error bar, S.E.M. ${ }^{* * \star} P<0.001$ compared with Ars. (c) The percentage of OP-puro puncta adjacent to/colocalizing with SGs is shown. Error bar, S.E.M. ${ }^{* \star *} P<0.001$ compared with Ars. (d) HeLa cells lipofected for $72 \mathrm{~h}$ with control, VCP, PLAA or UFD1L siRNA were treated for $45 \mathrm{~min}$ with OP-puro and Ars., fixed and stained with Alexa594-Azide, anti-TIA-1 and DAPI. Arrowheads indicate OP-puro-labeled DRIPs colocalizing with TIA-1-positive SGs. The percentage of OP-puro puncta adjacent to/colocalizing with SGs is shown. Error bar, S.E.M. ${ }^{* \star} P<0.001$ compared with cells transfected with control siRNA. (a, d) $2.5 \times$ magnification of the selected area. See also Supplementary Figure S5 
a
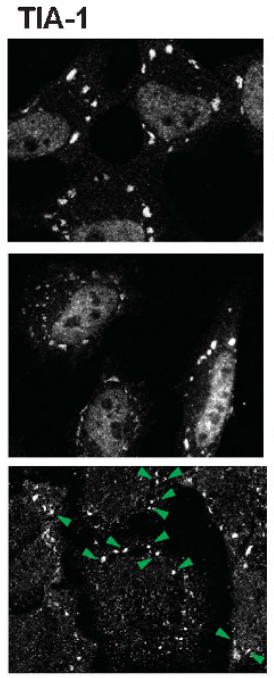

C G3BP
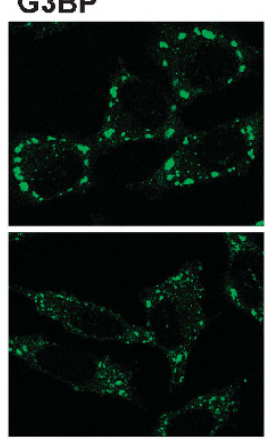

e
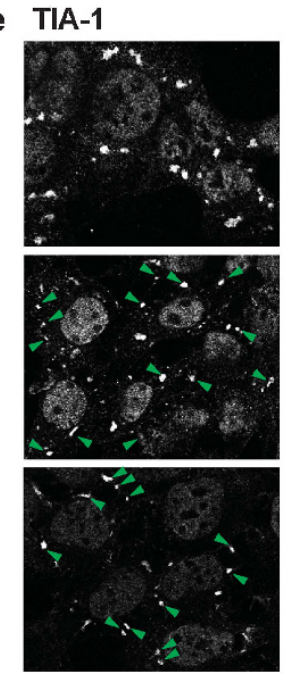

RPL19
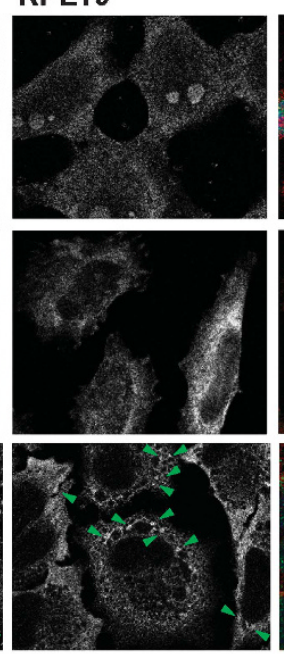

28S rRNA

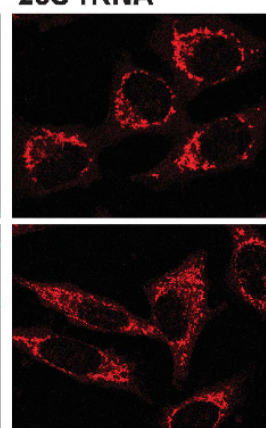

RPL19
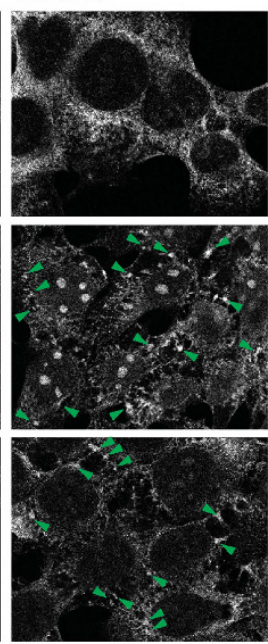

merge
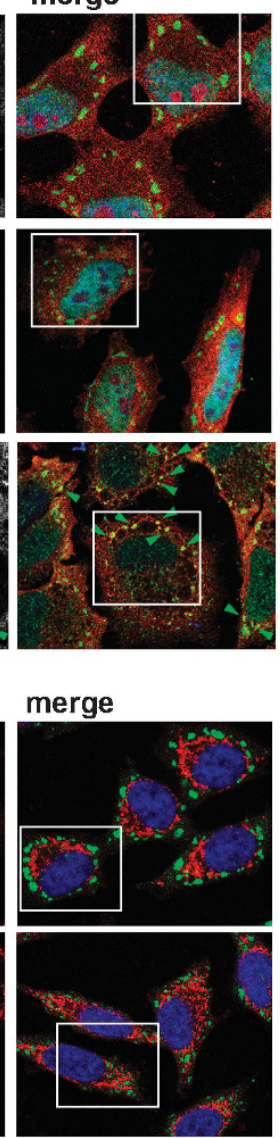

merge
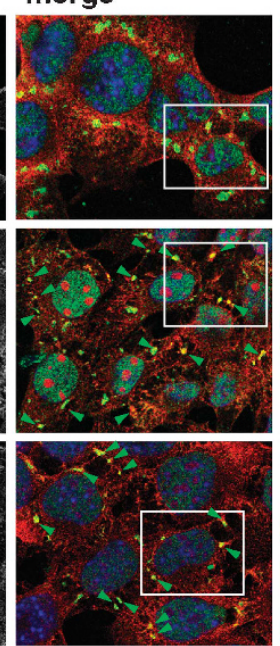

2.5x zoom
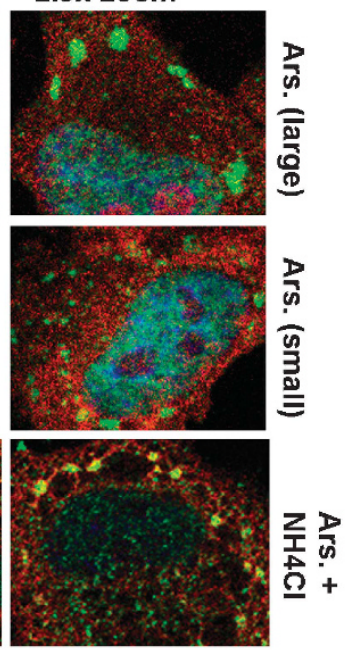

$2.5 \times$ zoom

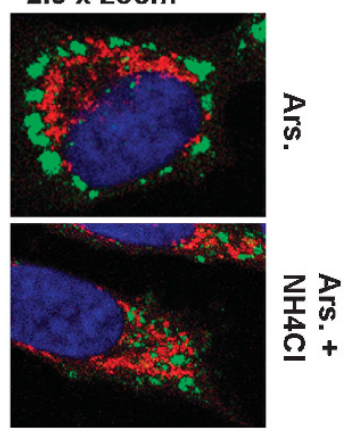

$2.5 \times$ zoom
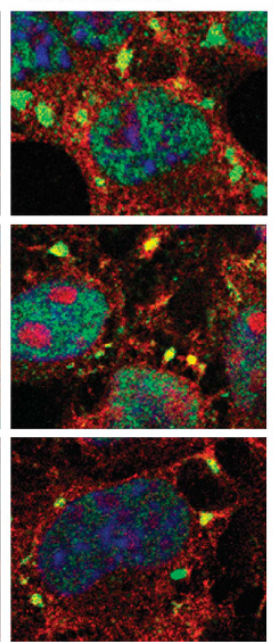

b

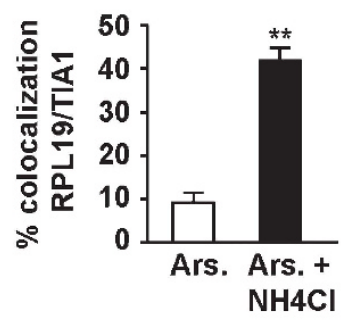

d
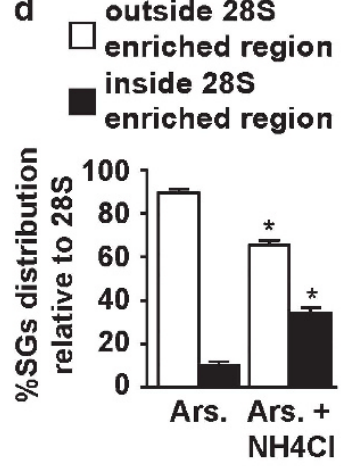

f

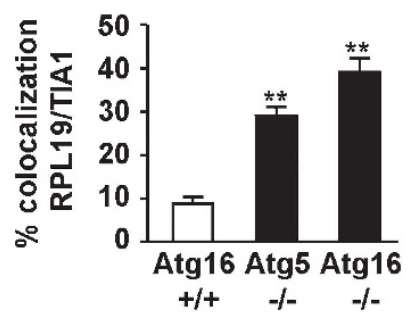

Figure 6 Chemical inhibition of lysosomes or genetic perturbation of autophagy leads to formation of SGs that contain RPL19. (a, b) HeLa cells were treated for 45 min with arsenite (Ars.) alone or following ammonium chloride $\left(\mathrm{NH}_{4} \mathrm{Cl}\right.$ ) pre-treatment. Cells were fixed and labeled with anti-TIA-1, RPL19 and DAPI. (b) Quantitation of colocalization of RPL19 and TIA-1 is shown. Error bar, S.E.M. ${ }^{*} P<0.01$. (c, d) Cells treated as described in a were fixed, subjected to in situ hybridization with a $28 \mathrm{~S}$ rRNA probe coupled to Alexa594 and labeled with anti-G3BP and DAPI. (d) Quantitation of the \% of TIA-1-positive SGs located outside or inside the 28S rRNA perinuclear-enriched region is shown. Error bar, S.E.M. ${ }^{*} P<0.05$. (e, f) Atg16 $6^{+1+}$, Atg5 ${ }^{-1-}$ and Atg16 $6^{-1-}$ MEFs were treated for 45 min with Ars. and labeled with anti-TIA-1, RPL19 and DAPI. (f) Quantitation of colocalization of RPL19 and TIA-1 is shown. Error bar, S.E.M. ${ }^{*} P<0.01$. (a-c) $2.5 \times$ magnification of the selected area. See also Supplementary Figure S6

Silencing VCP, PLAA and UFD1L also inhibited heat shock-induced SGs (Supplementary Figure S4A). Knockdown of VCP, PLAA or UFD1L did not induce Hsp70
(Supplementary Figure S4B) or apoptosis/PARP cleavage (Supplementary Figures S4B-D; PARP was slightly cleaved in VCP-depleted cells). As positive control, PARP cleavage 
was induced with doxorubicin and was blocked by cotreatment with zVAD-fmk (Supplementary Figure S4C). Likewise, co-treatment with ZVAD-fmk could not rescue the inhibitory effect of VCP or PLAA knockdown on SG assembly (data not shown). Finally, SG response was not affected in cells where ubiquitin-like-domain-containing protein Ubxd8 (Ubxd8), another VCP co-factor, was silenced (Supplementary Figures S4E-G). The VCP-Ubxd8 complex promotes the release of ubiquitinated HuR from ribonucleoprotein complexes; ${ }^{39} \mathrm{HuR}$ is a component of SGs but it is not required for their assembly. ${ }^{40}$ Thus, only a subset of VCP complexes affects SGs.

DRIPs are excluded but adjacent to SGs. To test whether accumulation of DRIPs occurs and correlates with decreased SG formation, we labeled nascent chains using O-propargyl-puromycin (OP-puro). ${ }^{25}$ Forty-five minutes after treatment, aggregated OP-puro-labeled DRIPs (stained with Alexa594-Azide) accumulated in the cytosol (Supplementary Figures S5A and B). Only a background Alexa594-Azide signal was detectable after co-treatment with OP-puro and cycloheximide, a translation inhibitor, demonstrating the specificity of OP-puro (Supplementary Figure S5C). While at the concentration and time used, OP-puro did not by itself induce SGs (Supplementary Figure S5B), concomitant treatment with arsenite and OP-puro induced SGs (Supplementary Figure S5D). DRIPs were excluded from SGs, but could be found adjacent to SGs (Supplementary Figure S5D, arrowheads). These OP-purolabeled products colocalized with ubiquitin (Supplementary Figure S5E) and the autophagy adaptor sequestosome 1 (SQSTM1; Supplementary Figure S5F), which can target certain ubiquitinated proteins, including DRIPs, to autophagosomes/lysosomes. ${ }^{41}$ Indeed, partial colocalization of DRIPs with LAMP2 (lysosomal associated membrane protein-2) was found after co-treatment with OP-puro and arsenite, supporting the fact that DRIPs released by polysomes can be targeted to lysosomes (Supplementary Figure S5G, arrowheads). Interestingly, while ubiquitin is a component of $\mathrm{SGs}^{8}$ and colocalizes with DRIPs adjacent to SGs (Supplementary Figures S5E and H), SQSTM1 only colocalized with nascent chains and was excluded from SGs (Supplementary Figures S5F and I). SQSTM1 adjacency to SGs further points to a possible link between extraction of ubiquitinated proteins, degradation systems and SG assembly. Inhibition of proteasome and/or lysosome significantly increased the number of OP-puro-labeled cytoplasmic puncta suggesting that DRIPs are targeted to proteasome and lysosome for disposal (Figures $5 \mathrm{a}$ and b). Bortezomib and $\mathrm{NH}_{4} \mathrm{Cl}$ also increased the adjacency of DRIPs to SGs (Figures $5 \mathrm{a}$ and $\mathrm{c}$ ). Thus, if not properly degraded, nascent peptides accumulate in the close vicinity of assembling SGs.

VCP, PLAA or UDF1L, which promote release and degradation of nascent proteins from the ribosome ${ }^{33}$ and which regulate SG assembly, colocalize with OP-puro-labeled peptides (Supplementary Figures S5J-L). While arseniteinduced SGs did not contain DRIPs in control cells (Figure 5d), OP-puro-labeled peptides significantly colocalized with TIA-1 in SGs in VCP-, PLAA- or UFD1L-deficient cells (Figure $5 \mathrm{~d}$ ). Thus, inhibition of lysosomes, VCP, PLAA and UFD1L leads to formation of altered SGs that are adjacent to/contain undigested DRIPs.

RPL19 is retained in SGs that form in cells with impaired autophagy, lysosome or VCP. Upon proteotoxic stress, polysomes disassemble and also release, besides DRIPs, $40 S$ and $60 S$ ribosomes. While $40 S$ are recruited into SGs (Supplementary Figure S6A), 60S are excluded from SGs and antagonize SG formation ${ }^{22,32}$ (Figure 6a). Nonfunctional 605 degradation is regulated by diverse processes, including VCP-UFD1 dissociation, ${ }^{42}$ which occurs following proteasome inhibition and treatment with arsenite; ${ }^{42,43}$ ribophagy, ${ }^{37}$ which requires four players that also modulate $S G$ assembly: VCP (Cdc48), PLAA (Ufd3), USP10 (Ubp3) and G3BP (Bre5) $^{13,14,37,38}$ (and our results; Figures 3 and 4); and lysosomes, which directly digest rRNA. ${ }^{44}$ Thus, we asked whether 605 accumulate adjacent to/within assembling SGs after autophagy, lysosome or VCP inhibition, analogous to what we observed with DRIPs. We assessed ribosomal protein L19 (RPL19) and 28S rRNA, both 60S components. RPL19 was devoid from both large and smaller SGs that accumulated in HeLa cells treated with arsenite, while it significantly colocalized with TIA-1 in SGs following cotreatment with arsenite and $\mathrm{NH}_{4} \mathrm{Cl}$ (Figures $6 \mathrm{a}$ and $\mathrm{b}$ ). In line with this, 28S rRNA was excluded from the large SGs induced by arsenite (Figure 6c), but showed a perinuclearenriched distribution with the SGs generally aligned outside this region. Following exposure to arsenite and $\mathrm{NH}_{4} \mathrm{Cl}$, we observed a more disorganized distribution of SGs that were smaller in size and accumulated in the $28 \mathrm{~S}$ rRNA perinuclear-enriched region (Figures $6 c$ and d). Similar to HeLa cells, arsenite-treated autophagy-proficient cells formed SGs that did not contain RPL19 (Figures 6e and f). Instead, RPL19 and TIA-1 colocalized in arsenite-induced SGs in Atg5 $5^{-1-}$ and Atg16 $^{-/-}$cells (Figures $6 e$ and f). In autophagy-proficient cells co-treated with arsenite and $\mathrm{NH}_{4} \mathrm{Cl}$, TIA-1 colocalization with RPL19 was similar to that seen in autophagy-deficient cells (Supplementary Figure S6C). Finally, VCP-depleted cells showed increased colocalization of RPL19 with TIA-1-positive SGs (Figures 7a and b) and displayed higher basal levels of RPL19 (Figure 7c). The spatial distribution of SGs and 28S rRNA was also affected in VCP-deficient cells, where SGs accumulated in the $28 \mathrm{~S}$ rRNA-enriched perinuclear region (Figures $7 d$ and $e$ ). Together, our results demonstrate that cells with inhibited autophagy, lysosome or VCP form SGs with altered morphology that retain 605 components within or in their close vicinity.

\section{Discussion}

The finding that SGs are targeted to autophagy for degradation by $\mathrm{VCP}^{15}$ and that SG disassembly requires chaperonedriven protein disaggregation ${ }^{45}$ are consistent with the idea that PQC may survey and/or assist SG dynamics, both assembly and disassembly. However, despite these observations, it was unclear whether the $P Q C$ actively participates in SG assembly and whether impairments thereof affect SGs.

Our data demonstrate that inhibition of autophagy, Iysosomes and VCP impairs SGs, supporting that the PQC 
a
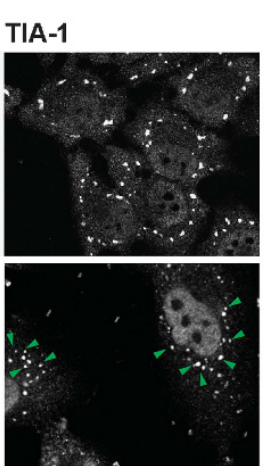

RPL19
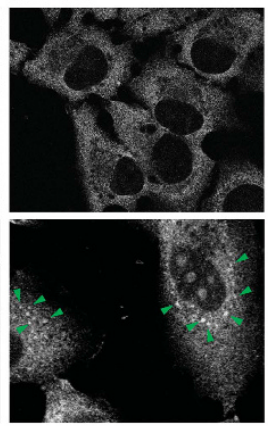

c

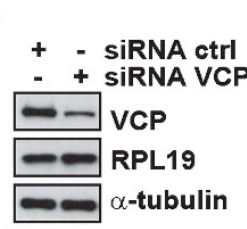

d G3BP
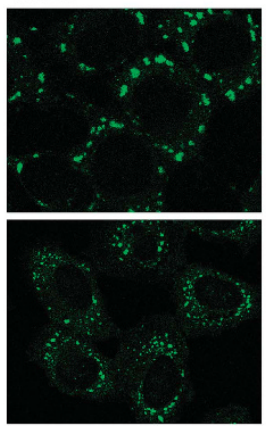

28S rRNA

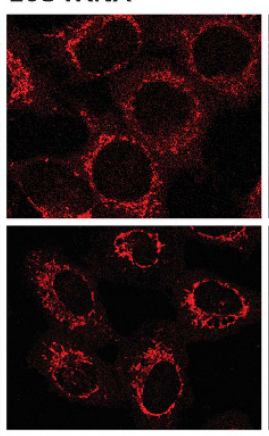

merge
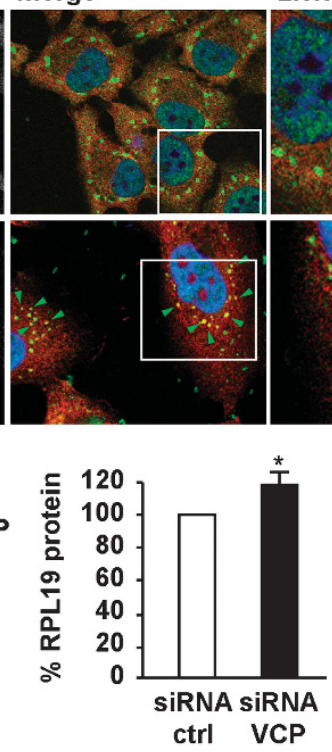

$2.5 \times \mathrm{zoom}$
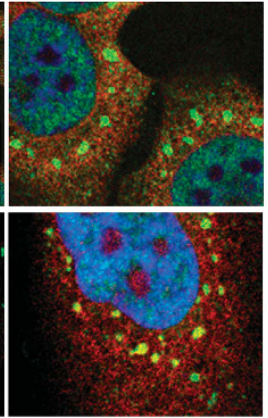

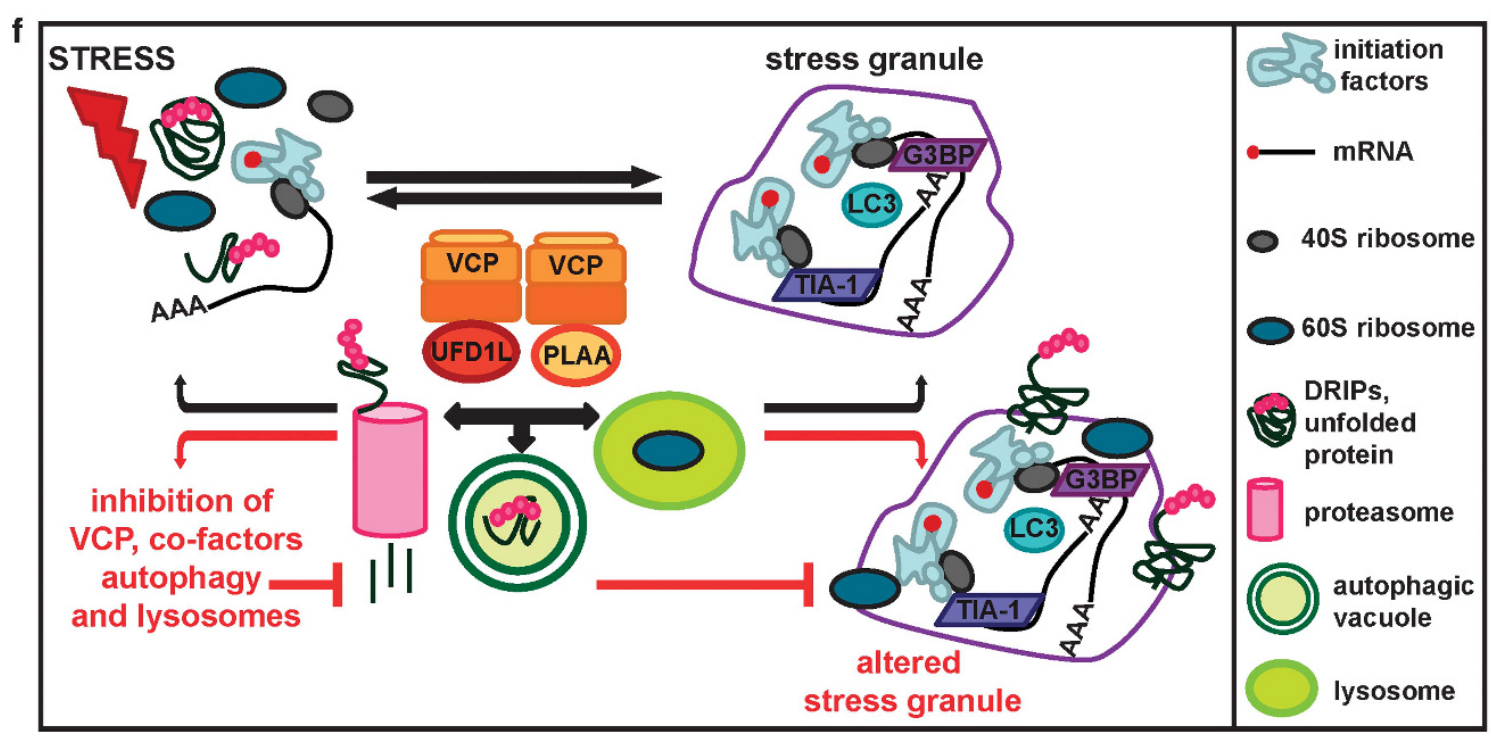

modulates SG formation. Autophagy, lysosomes and VCP govern protein (and organelle) degradation. In cells with impaired autophagy, lysosome or VCP function, SGs were smaller in size as compared with SGs forming in control cells.
This suggests that specific components may need to be extracted from the foci where SGs assemble to be targeted to degradation; this may indirectly contribute to optimal SG growth. DRIPs and 605 are released by disassembling 
Figure 7 RPL19 and 28S rRNA partly colocalize with arsenite-induced SGs in VCP-depleted cells. HeLa cells lipofected for $72 \mathrm{~h}$ with control or VCP siRNA were treated for 45 min with arsenite (Ars.), fixed and labeled with anti-TIA-1, RPL19 and DAPI (a, b) or subjected to western blot (c) or to in situ hybridization with a $28 \mathrm{~S}$ rRNA probe, followed by co-staining with anti-G3BP and DAPI (d, e). (b) Quantitation of colocalization of RPL19 and TIA-1 is shown. Error bar, S.E.M. ${ }^{* *} P<0.01$. (c) Quantitation of RPL19 protein levels is shown. Error bar, S.E.M. ${ }^{*} P<0.05$. (e) Quantitation of the \% of TIA-1-positive SGs located outside or inside the $28 \mathrm{~S}$ rRNA perinuclear-enriched region is shown. Error bar, S.E.M. ${ }^{*} P<0.05$. (f) Schematic model of the proposed interplay between VCP, autophagy and SGs. Upon stress polysomes disassemble releasing DRIPs and ribosome subunits (40S and 60S), while mRNA-binding proteins containing prion-like domains (e.g., TIA-1) trigger the sequestration of bound mRNAs into SGs. SGs also contain, besides initiation factors, 40 S and the autophagy protein LC3. Instead, DRIPs and 60S are excluded from assembled SGs. DRIPs and ubiquitinated unfolded proteins are targeted to degradation by proteasome and autolysosomes with the assistance of VCP and its co-factors UFD1L and PLAA. Also damaged 60S can be targeted to degradation by proteasome and/or lysosome, a process that also involves VCP and co-factors, while undamaged 60S can be recycled (not shown). Inhibition of autophagy or Iysosomes, as well as depletion of VCP (and the co-factors PLAA or UFD1L) leads to the accumulation of DRIPs and 60S within the foci where SGs are assembling. Under such conditions, the SG response is decreased (in term of SG size and number) and SGs contain the non-canonical components DRIPs and $60 \mathrm{~S}$. (a, d) $2.5 \times$ magnification of the selected area

polysomes, prior to SG assembly, and are among the clients that are cleared with the assistance of autophagy, lysosomes and VCP. ${ }^{3,4,18,22,33,46-48}$ We found that DRIPs are excluded from arsenite-induced SGs; however, upon UPS and lysosome inhibition, DRIPs accumulated adjacent to SGs. DRIPs also significantly colocalized with SGs in VCP-, UFD1- and PLAA-depleted cells, consistent with their role in handling and degradation of DRIPs. These results suggest that impaired extraction of DRIPs from the foci where SGs assemble may affect SG composition and morphology (Figure 7f).

Ribosomes are also released by disassembling polysomes. Only the $40 S$ is a component of SGs, while the $60 S$ is excluded and impedes SG assembly. ${ }^{22} 28 \mathrm{~S}$ rRNA is enriched in the perinuclear region and SGs form in the close vicinity of, but always outside, this region. Instead, upon autophagy, VCP or lysosomal inhibition, SGs tend to accumulate within the perinuclear 28S rRNA-enriched region. Moreover, RPL19 colocalized with SGs in cells with impaired autophagy, lysosome or VCP. Consistent with their role in ribophagy and SG assembly ${ }^{13,14,38}$ (and Figures 3 and 4), inhibition of VCP, autophagy or lysosome alters SG composition, morphology and 60S distribution. This suggests that VCP may participate in extracting 605 from the foci where SGs are assembling. 605 can be next recycled or, when damaged due to the proteotoxic stress that elicits SGs, targeted to degradation via ribophagy, or both (Figure 7f). To what extent 605 are degraded or recycled, allowing polysome reassembly, is currently unknown. Our findings that RPL19 was trapped within SGs in cells with inhibited autophagy/ lysosome and VCP suggest that autolysosome-based degradation of some 60 S occurs concomitantly to SG assembly. At this stage we cannot exclude that, besides DRIPs and 60S, other components accumulate within SGs with altered morphology that form in cells with proteostasis (autophagy, lysosome and VCP) dysfunction, and that such additional components may be major causes of the impaired SG assembly. An important challenge for future work will be to identify how VCP and co-factors orchestrate the selection of components to be extracted from SGs and understanding LC3 role in SGs.

The discovery that autophagy, lysosome and VCP inhibition affects SG morphology and composition implies that proteostasis imbalances will have a direct impact on SGs. This may render the cells vulnerable under challenging/disease conditions. Indeed, recent data implicate SGs and deregulated proteostasis in amyotrophic lateral sclerosis, frontotemporal lobar degeneration and multi- system proteinopathy, which are also associated with VCP mutations ${ }^{49-54}$ and where protein aggregates that contain SG components accumulate. Thus, inappropriate SG dynamics may be relevant to pathogenesis. Our data are in line with this hypothesis and demonstrate that SGs forming under conditions of autophagy/lysosome or VCP inhibition accumulate non-canonical components (DRIPs and 60S); this in turn, can impair SG dynamics and contribute to SG persistency. Persistent or partly disassembled SGs, if not properly disposed, ${ }^{15}$ may act as seeds for aggregation, further challenging protein and RNA homeostasis. In parallel, SGs sequester pro-apoptotic factors, indirectly inhibiting apoptosis. ${ }^{10,13,55}$ Although we could not detect any major sign of toxicity under our experimental conditions (in line with the findings from Buchan et al. ${ }^{15}$ ), we cannot exclude that, as a consequence of proteostasis imbalances, chronic-impaired SG formation may lead to, for example, deregulation of signaling pathways and decreased sequestration of pro-apoptotic factors, which could also contribute to cell vulnerability/death.

\section{Materials and Methods}

Cell culture, treatments and transfection. HeLa cells, Atg16 ${ }^{+1+}$, Atg16 $6^{-1-}, \operatorname{Atg} 5^{+1+}, A \operatorname{Atg} 5^{-1-}$ and m5-7 MEFs were cultured in DMEM (ECB7501L; EuroClone, Milan, Italy) supplemented with $2 \mathrm{mM}$ L-glutamine, $100 \mathrm{U} / \mathrm{ml}$ penicillin/streptomycin and $10 \%$ fetal bovine serum (Lonza, Basel, Switzerland) in a $37^{\circ} \mathrm{C}$ incubator with $5 \% \mathrm{CO}_{2}$.

$\mathrm{Atg}^{+1+}$ and $\mathrm{Atg} 5^{-1-}$ MEFs were kindly provided by Dr. T Yoshimori (Osaka University). Transfections were performed using Lipofectamine 2000 (Life Technologies, Monza, Italy) according to the manufacturer's instructions. All siRNAs used were from Dharmacon/GE Healthcare (Milan, Italy): siGENOME non-targeting control siRNA, ON-TARGETplus VCP siRNA, ON-TARGETplus PLAA siRNA, ON-TARGETplus UFD1L siRNA and ON-TARGETplus Ubxd8 siRNA.

Cells were treated with the following drugs at the concentrations indicated here: arsenite $0.5 \mathrm{mM}$ or, when specified, 0.1 mM; Z-Leu-Leu-Leu-al (MG132) $20 \mu \mathrm{M}$; ammonium chloride $\left(\mathrm{NH}_{4} \mathrm{Cl}\right) 20 \mathrm{mM}$; CLQ $50 \mu \mathrm{M}$; ML240 $5 \mu \mathrm{M}$; Eerl $10 \mu \mathrm{M}$; Bortezomib (Bort.) $100 \mathrm{nM}$; OP-puro $25 \mu \mathrm{M}$.

Immunofluorescence microscopy. Unless otherwise indicated, cells were grown on coverslip, treated as indicated, washed with cold PBS and fixed with $3.7 \%$ formaldehyde in PBS for $9 \mathrm{~min}$ at room temperature, followed by permeabilization with cold acetone for $5 \mathrm{~min}$ at $-20^{\circ} \mathrm{C}$. Blocking and incubation with primary and secondary antibodies were performed in PBS containing $3 \%$ BSA and $0.1 \%$ Triton $\mathrm{X}-100$. Primary and secondary antibodies used are listed below. Analysis of the cells was done by confocal imaging using a Leica SP2 AOBS system (Leica Microsystems, Milan, Italy) and a $63 \times$ oil-immersion lens.

OP-puro labeling of cultured cells. The protocol for OP-puro labeling was adapted from Liu et al. ${ }^{25}$ Cells were incubated with $25 \mu \mathrm{M}$ OP-puro for $45 \mathrm{~min}$ and CuAAC detection of OP-puro incorporated into nascent proteins was performed as previously described, using Alexa Fluor 594 azide (A10270, Life 
Technologies) (Liu J et al. ${ }^{25}$ ). Cells were next processed for immunofluorescence microscopy as described above.

In situ hybridization. The in situ hybridization protocol was adapted from Reineke et al. ${ }^{56}$ Cells were fixed with $2 \%$ formaldehyde in PBS for $1 \mathrm{~min}$ at room temperature and permeabilized with ice-cold methanol for $10 \mathrm{~min}$ at $-20^{\circ} \mathrm{C}$. The following $5^{\prime}$-biotinylated DNA probe was used to detect 28S rRNA: $5^{\prime}$-cggcgctgccgtatcgttccgcctggge gggattctgacttagaggcgttc-3'. The probe was hybridized in hybridization buffer (50\% formamide, $2 \times$ SSC, $10 \%$ dextran sulfate, $0.2 \%$ BSA, $5 \mathrm{mM} \mathrm{DTT}$ ) at a final concentration of $1 \mu \mathrm{g} / \mathrm{ml}$ for $24 \mathrm{~h}$ at $43^{\circ} \mathrm{C}$ in a humidified chamber and detected by subsequent incubation with Cy3 streptavidin (GE Healthcare Amersham, Milan, Italy) in $4 \times$ SSC plus $0.1 \%$ Triton $\mathrm{X}-100$ for $1 \mathrm{~h}$ at room temperature. Blocking and incubation with primary and secondary antibodies were next performed as described above.

Western blotting. Cells were lysed in Laemmli buffer and protein samples were boiled $3 \mathrm{~min}$ at $100^{\circ} \mathrm{C}$, separated by SDS-PAGE, transferred onto nitrocellulose membranes, subjected to western blot analysis, and visualized using an ECL detection kit (Thermo Scientific, Milan, Italy).

Antibodies and reagents. The primary antibodies used are listed. Mouse anti-LAMP2 (H4B4), mouse anti-PARP-1(F-2), mouse anti-PLAA (E-1), mouse anti-Ribosomal Protein L19 (K-12), mouse anti-SQSTM1 (D-3), mouse anti-UFD1 (19), rabbit anti-SQSTM1 (H-290), rabbit anti-ETEA (H-300) and goat anti-TIA1(C-20) were from Santa Cruz Biotechnology Inc. (Heidelberg, Germany). Mouse anti-G3BP and mouse anti-HSP70 (SMC-100A/B) were from BD Biosciences (Milan, Italy) and StressMarq Biosciences Inc., (Victoria, BC, Canada) respectively. Mouse anti-Ribosomal Protein $\mathrm{L} 19$ and mouse anti-VCP were from Abnova (Tapei, Taiwan) and Thermo Scientific, respectively. Mouse anti- $\alpha$-tubulin and rabbit anti-phospho elF2 $\alpha$ were from Sigma-Aldrich (Milan, Italy). Rabbit anti-LC3 (Novus Biologicals Ltd, Cambridge, UK) was used in the majority of our experiments, unless otherwise indicated.

The secondary antibodies used are listed. All the Alexa-conjugated secondary antibodies were from Life Technologies: donkey-anti-goat-Alexa488, donkey-antimouse-Alexa594, donkey-anti-rabbit-Alexa594, donkey-anti-rabbit-Alexa488 and donkey-anti-mouse-Alexa488. Mouse and rabbit HRP-conjugated secondary antibodies for western blot were from GE Healthcare Europe GmbH (Milan, Italy).

The reagents used in this study are as follows: ammonium chloride (A9434), chloroquine (C6628), OP-puro (P8833), sodium arsenite (Carlo Erba Reagents, Cornaredo, Italy), z-Leu-Leu-Leu-al (MG132; C2211), cycloheximide (C7698) and tetracycline hydrochloride (T7660) were from Sigma-Aldrich. Eerl (sc-358130) was from Santa Cruz Biotechnology, Inc. Bort. (S1013) was from Selleck Chemicals (Munich, Germany); ML240 was a kind gift from Prof. RJ Deshaies.

Statistics, analysis of SG size and quantification of colocalization. Student's $t$-test was used for comparisons between two groups. One-way ANOVA followed by Bonferroni-Holm post-hoc test was used for comparisons between three or more groups. ${ }^{\star} P<0.05 ;{ }^{\star \star} P<0.01 ;{ }^{* \star \star} P<0.001$.

SG size was measured using ImageJ software (http://rsb.info.nih.gov/ij). Average size of 300 SGs is reported.

Colocalization efficiency of RPL19 with TIA-1 in SGs was performed using Image J software (Image J, Colocalization Coloc 2, Manders' correlation). Region-ofInterest (ROI) were drawn around single cells. Background and threshold correction were applied for each ROI.

\section{Conflict of Interest}

The authors declare no conflict of interest.

Acknowledgements. We thank RJ Deshaies for providing ML240. SC is grateful to MIUR (Rita Levi Montalcini Principal Research Fellowship). SC and AP are grateful to AriSLA for funding. DCR is grateful to the Wellcome Trust (Principal Research Fellowship) for funding.

1. Bukau B, Weissman J, Horwich A. Molecular chaperones and protein quality control. Cell 2006; 125: 443-451.

2. Kim YE, Hipp MS, Bracher A, Hayer-Hartl M, Hartl FU. Molecular chaperone functions in protein folding and proteostasis. Annu Rev Biochem 2013; 82: 323-355.
3. Korolchuk VI, Mansilla A, Menzies FM, Rubinsztein DC. Autophagy inhibition compromises degradation of ubiquitin-proteasome pathway substrates. Mol Cell 2009; 33: 517-527.

4. Schubert U, Anton LC, Gibbs J, Norbury CC, Yewdell JW, Bennink JR. Rapid degradation of a large fraction of newly synthesized proteins by proteasomes. Nature 2000; 404 : 770-774.

5. Anderson P, Kedersha N. Stress granules. Curr Biol 2009; 19: R397-R398.

6. Gilks N, Kedersha N, Ayodele M, Shen L, Stoecklin G, Dember LM et al. Stress granule assembly is mediated by prion-like aggregation of TIA-1. Mol Biol Cell 2004; 15: 5383-5398.

7. Athanasopoulos V, Barker A, Yu D, Tan AH, Srivastava M, Contreras N et al. The ROQUIN family of proteins localizes to stress granules via the $\mathrm{ROQ}$ domain and binds target mRNAs. FEBS J 2010; 277: 2109-2127.

8. Kwon S, Zhang Y, Matthias P. The deacetylase HDAC6 is a novel critical component of stress granules involved in the stress response. Genes Dev 2007; 21: 3381-3394.

9. Ohn T, Kedersha N, Hickman T, Tisdale S, Anderson P. A functional RNAi screen links O-GIcNAc modification of ribosomal proteins to stress granule and processing body assembly. Nat Cell Biol 2008; 10: 1224-1231.

10. Kim WJ, Back SH, Kim V, Ryu I, Jang SK. Sequestration of TRAF2 into stress granules interrupts tumor necrosis factor signaling under stress conditions. Mol Cell Biol 2005; 25 . 2450-2462.

11. Mazroui R, Di Marco S, Kaufman RJ, Gallouzi IE. Inhibition of the ubiquitin-proteasome system induces stress granule formation. Mol Biol Cell 2007; 18: 2603-2618.

12. Kawaguchi Y, Kovacs JJ, McLaurin A, Vance JM, Ito A, Yao TP. The deacetylase HDAC6 regulates aggresome formation and cell viability in response to misfolded protein stress. Cell 2003; 115: 727-738.

13. Takahashi M, Higuchi M, Matsuki H, Yoshita M, Ohsawa T, Oie M et al. Stress granules inhibit apoptosis by reducing reactive oxygen species production. Mol Cell Biol 2013; 33: 815-829.

14. Tourriere H, Chebli K, Zekri L, Courselaud B, Blanchard JM, Bertrand E et al. The RasGAP-associated endoribonuclease G3BP assembles stress granules. J Cell Biol 2003; 160: 823-831.

15. Buchan JR, Kolaitis RM, Taylor JP, Parker R. Eukaryotic stress granules are cleared by autophagy and Cdc48/VCP function. Cell 2013; 153: 1461-1474.

16. Dargemont C, Ossareh-Nazari B. Cdc48/p97, a key actor in the interplay between autophagy and ubiquitin/proteasome catabolic pathways. Biochim Biophys Acta 2011; 1823: $138-144$

17. Ju JS, Fuentealba RA, Miller SE, Jackson E, Piwnica-Worms D, Baloh RH et al. Valosincontaining protein (VCP) is required for autophagy and is disrupted in VCP disease. J Cell Biol 2009; 187: 875-888.

18. Richly $H$, Rape M, Braun $S$, Rumpf $S$, Hoege $C$, Jentsch $S$. A series of ubiquitin binding factors connects $\mathrm{CDC} 48 / \mathrm{p} 97$ to substrate multiubiquitylation and proteasomal targeting Cell 2005; 120: 73-84.

19. Klionsky DJ, Codogno P. The mechanism and physiological function of macroautophagy. J Innate Immun 2013; 5: 427-433.

20. Piper RC, Katzmann DJ. Biogenesis and function of multivesicular bodies. Annu Rev Cell Dev Biol. 2007; 23: 519-547.

21. Kabeya Y, Mizushima N, Ueno T, Yamamoto A, Kirisako T, Noda T et al. LC3, a mammalian homologue of yeast Apg8p, is localized in autophagosome membranes after processing. EMBO J 2000; 19: 5720-5728.

22. Kedersha N, Cho MR, Li W, Yacono PW, Chen S, Gilks N et al. Dynamic shuttling of TIA-1 accompanies the recruitment of mRNA to mammalian stress granules. J Cell Biol 2000; 151: $1257-1268$.

23. Jacobson T, Navarrete C, Sharma SK, Sideri TC, Ibstedt S, Priya S et al. Arsenite interferes with protein folding and triggers formation of protein aggregates in yeast. $J$ Cell Sci 2012; 125(Pt 21): 5073-5083.

24. Anderson P, Kedersha N. Stress granules: the Tao of RNA triage. Trends Biochem Sci. 2008; 33: 141-150.

25. Liu J, Xu Y, Stoleru D, Salic A. Imaging protein synthesis in cells and tissues with an alkyne analog of puromycin. Proc Natl Acad Sci USA 2011; 109: 413-418.

26. Kuma A, Hatano M, Matsui M, Yamamoto A, Nakaya H, Yoshimori T et al. The role of autophagy during the early neonatal starvation period. Nature 2004; 432: 1032-1036.

27. Moreau K, Ravikumar B, Renna M, Puri C, Rubinsztein DC. Autophagosome precursor maturation requires homotypic fusion. Cell 2011; 146: 303-317.

28. Nishida Y, Arakawa S, Fujitani K, Yamaguchi H, Mizuta T, Kanaseki T et al. Discovery of Atg5/Atg7-independent alternative macroautophagy. Nature 2009; 461: 654-658.

29. Hosokawa N, Hara Y, Mizushima N. Generation of cell lines with tetracycline-regulated autophagy and a role for autophagy in controlling cell size. FEBS Lett 2007; 581: 2623-2629.

30. Farny NG, Kedersha NL, Silver PA. Metazoan stress granule assembly is mediated by P-elF2alpha-dependent and -independent mechanisms. RNA 2009; 15: 1814-1821.

31. Chou TF, Li K, Frankowski KJ, Schoenen FJ, Deshaies RJ. Structure-activity relationship study reveals ML240 and ML241 as potent and selective inhibitors of p97 ATPase. ChemMedChem 2013; 8: 297-312.

32. Kimball SR, Horetsky RL, Ron D, Jefferson LS, Harding HP. Mammalian stress granules represent sites of accumulation of stalled translation initiation complexes. Am J Physiol 2003; 284: C273-C284. 
33. Verma R, Oania RS, Kolawa NJ, Deshaies RJ. Cdc48/p97 promotes degradation of aberrant nascent polypeptides bound to the ribosome. ELife 2013; 2: e00308.

34. Seigneurin-Berny D, Verdel A, Curtet S, Lemercier C, Garin J, Rousseaux S et al. Identification of components of the murine histone deacetylase 6 complex: link between acetylation and ubiquitination signaling pathways. Mol Cell Biol 2001; 21: 8035-8044

35. Boyault C, Gilquin B, Zhang Y, Rybin V, Garman E, Meyer-Klaucke W et al. HDAC6-p97/ VCP controlled polyubiquitin chain turnover. EMBO J 2006; 25: 3357-3366.

36. Ju JS, Miller SE, Hanson PI, Weihl CC. Impaired protein aggregate handling and clearance underlie the pathogenesis of p97/VCP-associated disease. J Biol Chem 2008; 283 30289-30299.

37. Kraft C, Deplazes A, Sohrmann M, Peter M. Mature ribosomes are selectively degraded upon starvation by an autophagy pathway requiring the Ubp3p/Bre5p ubiquitin protease. Nat Cell Biol 2008; 10: 602-610.

38. Ossareh-Nazari B, Bonizec M, Cohen M, Dokudovskaya S, Delalande F, Schaeffer C et al. $\mathrm{Cdc48}$ and Ufd3, new partners of the ubiquitin protease Ubp3, are required for ribophagy. EMBO Rep 2010; 11: 548-554.

39. Zhou HL, Geng C, Luo G, Lou H. The p97-UBXD8 complex destabilizes mRNA by promoting release of ubiquitinated HuR from mRNP. Genes Dev 2013; 27: 1046-1058.

40. Lindquist ME, Lifland AW, Utley TJ, Santangelo PJ, Crowe Jr JE. Respiratory syncytia virus induces host RNA stress granules to facilitate viral replication. J Virol 2010; 84: 12274-12284

41. Clausen TH, Lamark T, Isakson P, Finley K, Larsen KB, Brech A et al. p62/SQSTM1 and ALFY interact to facilitate the formation of p62 bodies/ALIS and their degradation by autophagy. Autophagy 2010; 6: 330-344.

42. Fujii K, Kitabatake M, Sakata T, Ohno M. 40S subunit dissociation and proteasomedependent RNA degradation in nonfunctional 25S rRNA decay. EMBO J 2012; 31: 2579-2589.

43. Ding Q, Dimayuga E, Markesbery WR, Keller JN. Proteasome inhibition increases DNA and RNA oxidation in astrocyte and neuron cultures. J Neurochem 2004; 91: 1211-1218.

44. Saha BK, Graham MY, Schlessinger D. Acid ribonuclease from HeLa cell lysosomes. J Biol Chem 1979; 254: 5951-5957.

45. Cherkasov V, Hofmann S, Druffel-Augustin S, Mogk A, Tyedmers J, Stoecklin G et al. Coordination of translational control and protein homeostasis during severe heat stress. Curr Biol 2013; 23: 2452-2462.

46. Kirkin V, McEwan DG, Novak I, Dikic I. A role for ubiquitin in selective autophagy. Mol Cell 2009; 34: 259-269.

47. Meyer H, Bug M, Bremer S. Emerging functions of the VCP/p97 AAA-ATPase in the ubiquitin system. Nat Cell Biol 2012; 14: 117-123.
48. Defenouillere Q, Yao Y, Mouaikel J, Namane A, Galopier A, Decourty L et al Cdc48-associated complex bound to $60 \mathrm{~S}$ particles is required for the clearance of aberrant translation products. Proc Natl Acad Sci USA 2013; 110: 5046-5051.

49. Ju JS, Weihl CC. Inclusion body myopathy, Paget's disease of the bone and fronto-temporal dementia: a disorder of autophagy. Hum Mol Genet 2010; 19 : R38-R45.

50. Kim HJ, Kim NC, Wang YD, Scarborough EA, Moore J, Diaz Z et al. Mutations in prion-like domains in hnRNPA2B1 and hnRNPA1 cause multisystem proteinopathy and ALS. Nature 2013; 495: 467-473.

51. Li YR, King OD, Shorter J, Gitler AD. Stress granules as crucibles of ALS pathogenesis. J Cell Biol 2013; 201: 361-372.

52. Rodriguez-Ortiz CJ, Hoshino H, Cheng D, Liu-Yescevitz L, Blurton-Jones M, Wolozin B et al. Neuronal-specific overexpression of a mutant valosin-containing protein associated with IBMPFD promotes aberrant ubiquitin and TDP-43 accumulation and cognitive dysfunction in transgenic mice. Am J Pathol 2013; 183: 504-515.

53. Johnson JO, Mandrioli J, Benatar M, Abramzon Y, Van Deerlin VM, Trojanowski JQ et al. Exome sequencing reveals VCP mutations as a cause of familial ALS. Neuron 2010; 68: 857-864

54. Watts GD, Wymer J, Kovach MJ, Mehta SG, Mumm S, Darvish D et al. Inclusion body myopathy associated with Paget disease of bone and frontotemporal dementia is caused by mutant valosin-containing protein. Nat Genet 2004; 36: 377-381.

55. Thedieck K, Holzwarth B, Prentzell MT, Boehlke C, Klasener K, Ruf S et al. Inhibition of mTORC 1 by astrin and stress granules prevents apoptosis in cancer cells. Cell 2013; 154: 859-874.

56. Reineke LC, Dougherty JD, Pierre P, Lloyd RE. Large G3BP-induced granules trigger elF2 $\alpha$ phosphorylation. Mol Biol Cell 2012; 23: 3499-3510.

(i) This work is licensed under a Creative Commons Attribution 3.0 Unported License. The images or other third party material in this article are included in the article's Creative Commons license, unless indicated otherwise in the credit line; if the material is not included under the Creative Commons license, users will need to obtain permission from the license holder to reproduce the material. To view a copy of this license, visit http://creativecommons. org/licenses/by/3.0/

Supplementary Information accompanies this paper on Cell Death and Differentiation website (http://www.nature.com/cdd) 